\title{
Quasi-thermal noise spectroscopy: the art and the practice ${ }^{1}$
}

N. Meyer-Vernet, ${ }^{2}$ K. Issautier ${ }^{2}$, and M. Moncuquet $^{2}$

Corresponding author: N. Meyer-Vernet, LESIA, Observatoire de Paris. (nicole.meyer@obspm.fr)

${ }^{1}$ Dedicated to the memory of Jean-Louis

Steinberg

${ }^{2}$ LESIA, Observatoire de Paris, PSL

Research University, CNRS, Sorbonne

Univ., UPMC, Univ. Paris Diderot,

Sorbonne Paris Cité, 92195 Meudon, France

received 8 Jun, 2017, accepted 22 Jul 2017 


\section{Key Points.}

- We provide new calculations and analytical approximations for plasma measurements by QTN spectroscopy

- We study the compatibility with various space implementations and constraints

- We give some applications for future space missions

3 Abstract. Quasi-thermal noise spectroscopy is an efficient tool for mea-

4 suring in situ macroscopic plasma properties in space, using a passive wave

5 receiver at the ports of an electric antenna. This technique was pioneered

${ }_{6}$ on spinning spacecraft carrying very long dipole antennas in the interplan-

7 etary medium - like ISEE-3 and Ulysses - whose geometry approached a "the-

s oretician's dream". The technique has been extended to other instruments

9 in various types of plasmas onboard different spacecraft and will be imple-

10 mented on several missions in the near future. Such extensions require dif-

11 ferent theoretical modelizations, involving magnetized, drifting or dusty plas-

${ }_{12}$ mas with various particle velocity distributions, and antennas being shorter,

${ }_{13}$ biased or made of unequal wires. We give new analytical approximations of

${ }_{14}$ the plasma quasi-thermal noise (QTN), and study how the constraints of the

15 real world in space can (or cannot) be compatible with plasma detection by

${ }_{16}$ QTN spectroscopy. We consider applications to the missions Wind, Cassini,

${ }_{17}$ Bepi-Colombo, Solar Orbiter and Parker Solar Probe. 


\section{Introduction}

Thermal electromagnetic radiation, on which rely a large part of remote observations in astronomy and geophysics, is related to thermal fluctuations in radio-engineering circuits - the so-called Johnson noise - via the fluctuation-dissipation theorem. In the classical approximation, Nyquist's formula [Nyquist, 1928] tells us that a wave receiver in open circuit at the ports of an electric antenna immersed in black-body radiation of temperature $T$ measures a voltage power spectrum

$$
V_{f}^{2}=4 k_{B} T R
$$

${ }_{18}$ where $h f \ll k_{B} T$ ( $h$ being the Planck constant) and $R=R_{E M}$ is the antenna radiation

19 resistance (Figure 1, left). However most space missions involve electric antennas im-

20 mersed in plasmas (Figure 1, right), where the quasi-thermal motion of electric charges

${ }_{21}$ produces electrostatic fluctuations generally exceeding the radiation electromagnetic field.

${ }_{22}$ In that case the main contribution to the measured power is the plasma quasi-thermal

23 noise (QTN, Figure 2). This noise represents the long-wavelength measurement limit in

${ }_{24}$ radioastronomy [Meyer-Vernet et al., 2000] and it has been suggested to play a major

${ }_{25}$ role in the production of non-thermal electrons in the solar wind ([Yoon et al., 2016] and

${ }_{26}$ references therein).

${ }_{27}$ In the ideal case of a plasma at equilibrium temperature $T$, this noise reduces to

${ }_{28}$ Nyquist's formula (1) with $R=R_{P}$, the antenna resistance resulting from the plasma

${ }_{29}$ thermal fluctuations. If the plasma is non-thermal, the noise is still fully determined

so by the particle velocity distributions provided it is stable [e.g., Sitenko, 1967; Fejer and ${ }_{31}$ Kan, 1969]. This result can be generalized to a magnetized plasma and enables one to 
32 deduce the plasma properties from the measured voltage spectrum [Meyer-Vernet, 1979].

3з Since these electrostatic waves are significantly damped by the medium, the measured

${ }_{34}$ plasma properties are local ones, so that QTN spectroscopy provides in situ measure-

35 ments [e.g., Meyer-Vernet and Perche, 1989], contrary to the usual spectroscopy based

${ }_{36}$ on electromagnetic waves, which provides remote measurements.

This technique was pioneered aboard ISEE-3 which carried the most sensitive radio

${ }_{38}$ receiver ever flown [Knoll et al., 1978]. Rather ironically, the paper which pioneered

39 the technique [Meyer-Vernet, 1979], submitted ten days before the ISEE-3 launch, was

${ }_{40}$ in the process of being rejected on the grounds that the theory was too simple for being

${ }_{41}$ applicable in the solar wind, when the data of the inboard radio receiver became available;

${ }_{42}$ their agreement with the simple formulas proposed in the submitted manuscript prompted

${ }_{43}$ its immediate acceptance. This paper also provided a logically satisfying explanation for

${ }_{44}$ several observations previously interpreted as "new" emissions or instabilities, since “

${ }_{45}$ pluralitas non est ponenda sine necessitate" [Ockham, 1324]; the QTN explanation was

46 soon confirmed by Hoang et al. [1980] and Sentman et al. [1982].

${ }_{47} \quad$ The QTN measurement technique was subsequently used in various environments using

${ }_{48}$ radio receivers that had similarly not been designed for that purpose [e.g., Meyer-Vernet

49 et al., 1998]. In particular for measuring on ISEE-3/ICE the electron density and tem-

50 perature in a comet's tail [Meyer-Vernet et al., 1986a, b], where the electrons were too

${ }_{51}$ cold for the inboard particle analyzer to measure them accurately. The QTN technique

${ }_{52}$ was also used to measure the solar wind electron properties as a function of heliocentric

${ }_{53}$ distance [Hoang et al., 1992] and outside the ecliptic on Ulysses [e.g., Issautier et al.,

${ }_{54}$ 1998, 1999, 2008; Le Chat et al., 2011], and at 1 AU on WIND [e.g., Salem et al., 2001; 
${ }_{55}$ Issautier et al., 2005]. And also in planetary environments such as the Earth's outer

${ }_{56}$ plasmasphere [Lund et al., 1994], the Io plasma torus [e.g., Meyer-Vernet et al., 1993;

57 Moncuquet et al., 1995, 1997], and Saturn's magnetosphere [e.g., Moncuquet et al., 2005;

${ }_{58}$ Schippers et al., 2013] using the RPWS experiment on Cassini [Gurnett et al., 2004].

${ }_{59}$ Why is the QTN technique so well adapted to measure the electron density and temper-

${ }_{60}$ ature? There are four reasons for that. First of all, both properties are revealed in situ by

${ }_{61}$ the location and broad spectral shape of the plasma frequency peak (see Figure 2), just

${ }_{62}$ as traditional spectroscopy reveals the chemical composition and the temperature (albeit

${ }_{63}$ remotely). Second, being passive, this instrument does not perturb the medium, contrary

${ }_{64}$ to other wave techniques. Third, since it is based on electrostatic waves or fluctuations of

${ }_{65}$ wavelength of the order or greater than the Debye length (or the electron gyroradius if the

${ }_{66}$ plasma is strongly magnetised) and tending to infinity close to resonances, the technique

${ }_{67}$ is equivalent to a detector of cross-section larger by several orders of magnitude than

${ }_{68}$ that of classical detectors. And finally, for the same reason, it is relatively immune to

${ }_{69}$ spacecraft photoelectrons and charging effects which affect traditional particle analyzers;

70 in particular, since the electron density is deduced from a spectral peak, this measure-

${ }_{71}$ ment is independent on gain calibrations. Because of its reliability and accuracy, QTN

72 spectroscopy serves routinely to calibrate other instruments [e.g., Maksimovic et al., 1995;

73 Issautier et al., 2001; Salem et al., 2001].

${ }_{74}$ The drawback is that, contrary to the classical particle analysers, QTN spectroscopy

${ }_{75}$ cannot measure directly the particle velocity distributions. Even though some moments

76 are revealed by spectral features (see Section 2), a full measurement requires solving an

77 inverse problem: modelise the electric antenna and the velocity distribution(s) with a 
${ }_{78}$ few parameters, calculate the corresponding QTN spectrum, and fitting the theory to the

79 data to determine the parameters of the distribution as sketched in Figure 2. In other

so words, the QTN technique has the cons and pros of a global measurement: it measures

${ }_{81}$ less parameters, but it can measure them faster and more accurately. Note, too, that

82 the technique is less adapted to measure the ions because they are revealed at lower

${ }_{83}$ frequencies (Section 2.8) at which the spectrum can be spoiled by the shot noise.

${ }_{84}$ This shot noise, produced by the fluctuations due to collection and emission of individual

${ }_{85}$ electric charges by the antenna surface, can be a real nuisance for QTN spectroscopy. It is

${ }_{86}$ very hard to modelize because, contrary to the QTN, it depends on the antenna floating

${ }_{87}$ potential, which is badly known because the photoelectron and secondary emissions of

s materials in space change significantly with ageing and have different properties from

s9 those measured in the laboratory [e.g., Kawasaki et al., 2016]. This shot noise is generally

90 negligible for wire dipole antennas around $f_{p}$ [Meyer-Vernet and Perche, 1989], but this is

${ }_{91}$ not so when the antennas are made of small spheres. Indeed, the shot noise is proportional

${ }_{92}$ to the squared voltage produced by each charge collected or emitted $\left(\propto a^{-2}\right.$ for spheres

${ }_{93}$ of radius $a$ since their capacitance $\propto a$ ), and to the events' rate - proportional to surface

${ }_{94}$ area $\left(\propto a^{2}\right)$, so that the variation with $a$ cancels out. Therefore, the shot noise on spheres

${ }_{95}$ does not decrease as their radius decreases, contrary to wires whose surface $\propto a$ whereas

${ }_{96}$ the capacitance varies weakly with radius. This is the basic reason why spherical probes

${ }_{97}$ are unadaptated for QTN spectroscopy, in addition to the fact that these probes must be

${ }_{98} \quad$ supported by difficult-to-modelize booms. For all these reasons, we will only consider wire

99 antennas in this paper, and will mention the shot noise only for estimating the extent to 


\section{The Art}


${ }_{122}$ by their mutual coupling. At time scales corresponding to frequencies $f<f_{p}$, this dressing

${ }_{123}$ takes the simple form of a Debye sheath of scale $L_{D}$, the Debye length, so that each thermal ${ }_{124}$ electron produces on the antenna a voltage pulse of duration roughly equal to the time ${ }_{125}$ that it remains within a Debye length, i.e. about $1 /\left(2 \pi f_{p}\right)$; the Fourier transform of such ${ }_{126}$ a pulse is a constant for $f<f_{p}$, producing a plateau of amplitude determined by the bulk of the electrons. In contrast at higher frequencies, moving electrons excite plasma waves ${ }_{128}$ so that their dresses become more sophisticated [e.g., Meyer-Vernet, 1993], trailing long ${ }_{129}$ trains of Langmuir waves which produce the plasma frequency peak.

\subsection{Basics}

In the Vlasov framework, the plasma can be thought of as an assembly of independent test particles "dressed" by their collective interactions which determine the plasma dielectric permittivity defining the plasma spatial and temporal dispersion [Rostoker, 1961]. In vector and $c$ the speed of light), the (linear) longitudinal (E $\| \mathbf{k}$ ) electric field fluctuations

${ }_{135}$ in Fourier space are given from Poisson's equation by [Sitenko, 1967]

$$
\left\langle E^{2}(\mathbf{k}, \omega)\right\rangle=\frac{\left\langle\rho^{2}(\mathbf{k}, \omega)\right\rangle^{(0)}}{k^{2} \epsilon_{0}^{2}\left|\epsilon_{L}(\mathbf{k}, \omega)\right|^{2}}
$$

${ }_{140}$ bution $f(\mathbf{v})$ 


$$
\left\langle\rho^{2}(\mathbf{k}, \omega)\right\rangle^{(0)}=2 \pi e^{2} \int d^{3} v f(\mathbf{v}) \delta(\omega-\mathbf{k} \cdot \mathbf{v})
$$

${ }_{141}$ the particle number density being

$$
n=\int d^{3} v f(\mathbf{v})
$$

${ }_{142}$ In the presence of a magnetic field $\mathbf{B}$, the test particles follow helical orbits of (angular) ${ }_{143}$ gyrofrequency $\omega_{g}$, so that

$$
\left\langle\rho^{2}(\mathbf{k}, \omega)\right\rangle^{(0)}=2 \pi e^{2} \sum_{-\infty}^{\infty} \int d^{3} v f(\mathbf{v}) J_{n}^{2}\left(k_{\perp} v_{\perp} / \omega_{g}\right) \delta\left(\omega-n \omega_{g}-k_{\|} v_{\|}\right)
$$

${ }_{144}$ where $v_{\|}$and $v_{\perp}$ are the velocity components respectively parallel and perpendicular to B and $J_{n}$ are $n^{t h}$ order Bessel functions of the first kind [Abramowitz and Stegun, 1965].

With an electric antenna characterized by the current distribution $\mathbf{J}(\mathbf{k})$ in Fourier space, immersed in a plasma drifting with velocity $\mathbf{V}$, the voltage power at the antenna ports at

${ }_{148}$ frequency $f$ is

$$
V_{f}^{2}=\frac{2}{(2 \pi)^{3}} \int d^{3} k \frac{|\mathbf{k} . \mathbf{J}|^{2}}{k^{2}}\left\langle E^{2}(\mathbf{k}, \omega-\mathbf{k} \cdot \mathbf{V})\right\rangle
$$

${ }_{149}$ The power $V_{r}^{2}$ at the ports of a receiver of impedance $Z_{r}$ is deduced from

$$
V_{r}^{2} / V_{f}^{2}=\left|Z_{r} /\left(Z_{r}+Z_{a}\right)^{2}\right|
$$

where $Z_{a}$ is the antenna impedance. 


\subsection{Electric Antenna Response}

For the simplest antenna, made of two aligned wires, each of length $L \ll \lambda$ and radius $a \ll\left[L_{D}, L\right]$ (Figure 1), the current distribution can be assumed to be triangular $[$ Meyer and Vernet, 1974], so that

$$
|\mathbf{k} . \mathbf{J}|=\left|\frac{4 \sin ^{2}\left(k_{\|} L / 2\right)}{k_{\|} L} J_{0}\left(k_{\perp} a\right)\right|
$$

${ }_{154}$ where $k_{\|}$and $k_{\perp}$ are the $\mathbf{k}$ components respectively parallel and perpendicular to the antenna direction (see details in [Schiff, 1970; Couturier et al., 1981]). In most cases of interest, the wave numbers responsible for the noise are smaller than or of the order of the plasma Debye length (or the electron gyroradius if it is smaller), with $k a \ll 1$, so that $J_{0}\left(k_{\perp} a\right) \simeq 1$ except in very dense and cold plasmas as planetary ionospheres. An important consequence emerges from (8). Writing $k_{\|} L=k L \cos \alpha$ where $\alpha$ is the angle between $\mathbf{k}$ and the antenna direction, one sees that whereas a short antenna $(k L \ll 1)$ is mainly sensitive to $\mathbf{k}$ parallel to the antenna $(\cos \alpha=1)$ as for electromagnetic waves, on the contrary a long antenna $(k L \gg 1)$ is mainly sensitive to wave vectors roughly perpendicular to its proper direction [Meyer-Vernet, 1994].

If the plasma fluctuations are isotropic in the antenna frame (which holds with $V=0$ and an isotropic velocity distribution in a weakly magnetized plasma), (6) becomes

$$
V_{f}^{2}=\frac{8}{\pi^{2}} \int_{0}^{\infty} d k F(k L)\left\langle E^{2}(k, \omega)\right\rangle
$$

with 


$$
F(x)=1 /(32 \pi) \int d \Omega|\mathbf{k} . \mathbf{J}|^{2}=\left[\operatorname{Si}(x)-\operatorname{Si}(2 x) / 2-2 \sin ^{4}(x / 2) / x\right] J_{0}^{2}(x a / L) / x
$$

167

$F(x) \simeq x^{2} / 24$ for $x<1$

$F(x) \simeq \pi /(4 x)$ for $x \gg 1$

$$
V_{f}^{2}=\frac{1}{2 \pi^{2}} \int_{0}^{\infty} d k \int_{0}^{\pi} \sin \theta d \theta\left\langle E^{2}(k, \theta, \omega-k . V \cos \theta)\right\rangle \int_{0}^{2 \pi} \frac{d \phi}{2 \pi}|\mathbf{k} . \mathbf{J}|^{2}
$$

${ }_{174}$ where $\theta$ is the angle between $\mathbf{k}$ and the symmetry axis and (8) yields

$$
\int_{0}^{2 \pi} \frac{d \phi}{2 \pi}|\mathbf{k} . \mathbf{J}|^{2}=\frac{8}{\pi} \int_{0}^{2 \pi} d \phi \frac{\sin ^{4}(k L \cos \alpha / 2)}{(k L \cos \alpha)^{2}} J_{0}^{2}(k a \sin \alpha)
$$

${ }_{175} \alpha$ being the angle between $\mathbf{k}$ and the antenna direction, given by

$$
\cos \alpha=\cos \theta \cos \beta+\sin \theta \sin \beta \cos \phi
$$

${ }_{176}$ where $\beta$ is the angle between the antenna and the symmetry axis. 
If the antenna is parallel to the symmetry axis $(\beta=0)$, the QTN is given by (13) with ${ }_{178}$ from $(8)$

$$
\int_{0}^{2 \pi} \frac{d \phi}{2 \pi}|\mathbf{k} . \mathbf{J}|^{2}=\left[\frac{4 \sin ^{2}(k L \cos \theta / 2)}{|k L \cos \theta|} J_{0}(k a \sin \theta)\right]^{2}
$$

179 On the other hand, if the antenna is perpendicular to the symmetry axis $(\beta=\pi / 2)$, reduces to $\cos \alpha=\sin \theta \cos \phi$, so that with the change of variable $s=k L \sin \theta \cos \phi$ in the integral (14), we find

$$
\begin{aligned}
\int_{0}^{2 \pi} \frac{d \phi}{2 \pi}|\mathbf{k} . \mathbf{J}|^{2} & =F_{\perp}(k L \sin \theta) / 2 \\
F_{\perp}(x) & =\frac{64}{\pi} \int_{0}^{x} d s \frac{\sin ^{4}(s / 2)}{s^{2}\left(x^{2}-s^{2}\right)^{1 / 2}} \\
& =\frac{8}{x}\left[2 \int_{0}^{x} d t J_{0}(t)-\int_{0}^{2 x} d t J_{0}(t)+J_{1}(2 x)-2 J_{1}(x)\right]
\end{aligned}
$$

${ }_{182}$ where we have assumed $k a \ll 1$. Equation (19) yields $F_{\perp}(x) \simeq x^{2}$ for $x<1$, and ${ }_{183} \quad F_{\perp}(x) \simeq 8 / x$ for $x \gg 1$.

\subsection{Dealing with Non-Maxwellians: Generalized Temperatures}

Non-maxwellian velocity distributions are ubiquitous in space plasmas. The culprits are Coulomb collisions, whose cross-section decreases as the inverse square of the particle 


$$
\begin{aligned}
k_{B} T_{q} / m & =\left(\left\langle v^{q}\right\rangle / c_{q}\right)^{2 / q} & \\
c_{q} & =(q+1) ! ! & \text { for } q \text { even } \\
c_{q} & =\frac{2^{1+q / 2}}{\pi^{1 / 2}}\left(\frac{q+1}{2}\right) ! & \text { for } q \text { odd }
\end{aligned}
$$

${ }_{207}$ where $q>-3$ is an integer, $m$ is the electron mass, $k_{B}$ is Boltzmann's constant, and the ${ }_{208}$ scalar moment of order $q$ is 


$$
\left\langle v^{q}\right\rangle=\int d^{3} v v^{q} f(\mathbf{v}) / n
$$

${ }_{209}$ The coefficients $c_{q}$ are defined so that if the distribution is Maxwellian, all $T_{q}$ 's are equal ${ }_{210}$ to its classical temperature. The smaller the index $q$, the slower the particles responsible ${ }_{211}$ for $T_{q}$, and for velocity distributions having a suprathermal tail, the smaller the value of ${ }_{212} T_{q}$. In particular

$$
T_{2}=m\left\langle v^{2}\right\rangle /\left(3 k_{B}\right) \equiv T
$$

${ }_{213}$ is the classical kinetic temperature, $T_{1}$ is related to the mean random speed $\langle v\rangle$ as

$$
\langle v\rangle=\left[8 k_{B} T_{1} /(\pi m)\right]^{1 / 2}
$$

${ }^{214}$ and $T_{-2}$ is related to the Debye length $L_{D}$ as

$$
L_{D}=\left[\epsilon_{0} k_{B} T_{-2} /\left(n e^{2}\right)\right]^{1 / 2}
$$

${ }_{215}$ Therefore an instrument detecting essentially the low-energy particles (which determine ${ }_{216}$ the Debye length), will find, if a Maxwellian is assumed, a temperature close to $T_{-2}$, ${ }_{217}$ whereas an instrument measuring the flux will find a temperature close to $T_{1}$. It is there${ }_{218}$ fore not surprising that a number of temperature measurements in which a Maxwellian ${ }_{219}$ is assumed are inconsistent, so that new methods are being devised ([e.g., Dudik et al., ${ }_{220} 2017$ ] and references therein).

${ }_{221}$ The simplest way of representing a distribution having a supra-thermal tail is the so${ }_{222}$ called kappa distribution [Vasyliunas, 1968], which can be written 


$$
f_{\kappa}(v) \propto\left[1+v^{2} /\left(\kappa v_{0}^{2}\right)\right]^{-(\kappa+1)}
$$

${ }_{223}$ and has been used for modelling the QTN by Chateau and Meyer-Vernet [1991]; Zouganelis ${ }_{224}$ et al. [2008]; Le Chat et al. [2009]. Since the probability for the speed to lie in the range ${ }_{225}[v, v+d v]$ is $f_{\kappa}(v) \times 4 \pi v^{2} d v$ and we have $\left[\frac{d}{d v}\left[v^{2} f_{\kappa}(v)\right]\right]_{v=v_{0}}=0$, the most probable speed ${ }_{226}$ equals $v_{0}$. The greater the value of $\kappa$, the closer is the distribution to a Maxwellian, with ${ }_{227} \quad f_{\kappa}(v) \rightarrow e^{-v^{2} / v_{0}^{2}}$ when $\kappa \rightarrow \infty$.

At low speeds, developing $(27)$ in series yields $f_{\kappa}(v) \propto 1-(1+1 / \kappa) v^{2} / v_{0}^{2}$; hence the Kappa distribution decreases faster with $v$ than the Maxwellian $e^{-v^{2} / v_{0}^{2}} \propto 1-v^{2} / v_{0}^{2}$. In contrast, at high speeds $f_{\kappa}(v) \propto\left(v^{2} / \kappa v_{0}^{2}\right)^{-(\kappa+1)}$; hence the Kappa distribution decreases slower than a Maxwellian. This illustrates an interesting property of Kappa distributions.

$$
\begin{aligned}
T_{2} & =\left(m v_{0}^{2} / 2 k_{B}\right) \kappa /(\kappa-3 / 2) \equiv T \\
T_{1} & =T \times(\kappa-3 / 2)[\Gamma(\kappa-1) / \Gamma(\kappa-1 / 2)]^{2} \\
T_{-1} & =T \times(\kappa-3 / 2)[\Gamma(\kappa-1 / 2) / \Gamma(\kappa)]^{2} \\
T_{-2} & =T \times(\kappa-3 / 2) /(\kappa-1 / 2)
\end{aligned}
$$


${ }_{240}$ is given by (26), (28), and (31) as [Chateau and Meyer-Vernet, 1991]

${ }^{254}$ have as previously $T_{-1} \simeq T_{-2} \simeq T_{c}$.

\subsection{Core Electron Temperature}

$$
L_{D}=\frac{v_{0}}{\omega_{p}}\left[\frac{\kappa}{2 \kappa-1}\right]^{1 / 2}
$$
despite some arguments to the contrary [e.g., Fahr and Heyl, 2016]. $T_{h} / T_{c} \gg 1$, we have $T_{-1} \simeq T_{-2} \simeq T_{c}$, the core temperature.

suggesting that the Debye screening has a normal behavior even when $\kappa$ approaches $3 / 2$,

Another popular representation of non-thermal distributions is the sum of a cold ("core") and a hot maxwellian of respective density and temperature $n_{c}, n_{h}, T_{c}, T_{h}$, which has one more free parameter than the Kappa distribution. In that case we have $T \equiv T_{2}=\left(n_{c} T_{c}+n_{h} T_{h}\right) /\left(n_{c}+n_{h}\right)$, whereas $T_{-1}=T_{c}\left(n_{c}+n_{h}\right)^{2} /\left[n_{c}+n_{h}\left(T_{c} / T_{h}\right)^{1 / 2}\right]^{2}$ and $T_{-2}=\left(n_{c}+n_{h}\right) /\left(n_{c} / T_{c}+n_{h} / T_{h}\right)$; hence with a dilute hot maxwellian $\left(n_{h} / n_{c} \ll 1\right.$,

A further popular representation is the sum of a cold Maxwellian (of temperature $T_{c}$ ) ontaining the bulk of the distribution plus a hot Kappa distribution. Indeed, low-energy particles are generally collisional whereas faster ones are not, and many processes - inluding the spontaneously emitted Langmuir waves, i.e. the QTN [e.g., Yoon, 2014; Yoon et al., 2016] - tend to generate Kappa distributions via non-linearities. In that case, we As noted above, in a weakly magnetized plasma, the electron QTN spectrum exhibits a generic low-frequency plateau which is produced by electrons passing-by the antenna. This suggests that the plateau will mainly reveal the temperature defining the Debye length. 
${ }_{258}$ We derive below a generic expression of this plateau, relevant for a number of space

259 radio instruments and independent of the detailed shape of the distribution, provided it 260 is isotropic.

${ }_{261}$ In a weakly magnetized plasma with an isotropic electron velocity distribution, (3) 262 reduces to

$$
\left\langle\rho^{2}(\mathbf{k}, \omega)\right\rangle^{(0)}=\frac{(2 \pi e)^{2}}{k} \int_{\omega / k}^{\infty} d v v f(v)
$$

${ }_{263}$ which yields for $\omega / k v \ll 1$

$$
\left\langle\rho^{2}(\mathbf{k}, \omega)\right\rangle^{(0)} \simeq \frac{\pi e^{2}}{k} n\left\langle v^{-1}\right\rangle
$$

${ }_{264}$ In the same limit, we have

$$
\epsilon_{L} \simeq 1+\omega_{p}^{2}\left\langle v^{-2}\right\rangle / k^{2} \equiv 1+1 /\left(k^{2} L_{D}^{2}\right)
$$

${ }_{265}$ with $L_{D}$ given by (26). For a wire antenna (Figure 1), Eqs.(2), (9), (34) and (35) yield

$$
\begin{aligned}
V_{f}^{2} & \simeq\left(\frac{2^{7} m k_{B} T_{-2}^{2}}{\pi^{3} \epsilon_{0}^{2} T_{-1}}\right)^{1 / 2} F_{0}\left(L / L_{D}\right) \\
F_{0}(t) & =\int_{0}^{\infty} d y \frac{y F(y t)}{\left(1+y^{2}\right)^{2}}
\end{aligned}
$$

${ }_{266} F(x)$ being given by (10). The function $F_{0}\left(L / L_{D}\right)$ is shown in Figure 4 . The simple ${ }_{267}$ expression (36) of the plateau level is generic since it holds whatever the ratio $L / L_{D}$ and ${ }_{268}$ the shape of the (isotropic) electron velocity distribution.

For $L / L_{D} \gg 1, F(x)$ can be approximated by (12), so that (37) yields

$$
F_{0}\left(L / L_{D}\right) \simeq\left(\pi^{2} / 16\right) L_{D} / L \text { for } L / L_{D} \gg 1
$$


whence from (36)

$$
V_{f}^{2} \simeq \frac{(\pi / 2)^{1 / 2}}{\epsilon_{0} \omega_{p} L} \frac{k_{B} T_{-2}^{3 / 2}}{T_{-1}^{1 / 2}} \simeq \frac{3.5 \times 10^{-14}}{n^{1 / 2} L} \frac{T_{-2}^{3 / 2}}{T_{-1}^{1 / 2}} \text { for } L / L_{D} \gg 1
$$

${ }_{269}$ equivalent to a result by Chateau and Meyer-Vernet [1991]. One sees on Figure 4 that 270 the approximation (38) (dashed red line), yielding (39), only holds for extremely long ${ }_{271}$ antennas. In practice, however, one expects $L / L_{D} \sim 2.5-6$ for Bepi-Colombo and ${ }_{272} L / L_{D} \sim 1-2.5$ for Solar Orbiter in the solar wind at 0.3 AU,, whereas for Parker Solar ${ }_{273}$ Probe at 10 solar radii we have $L / L_{D} \sim 2-3$

274 In these cases, a much better approximation can be derived. Indeed, for $2 \lesssim L / L_{D} \lesssim 7$, ${ }_{275}$ (37) yields $F_{0} \simeq 0.05$ within $10 \%$ (solid red line in Figure 4 ), which yields

$$
V_{f}^{2} \simeq \frac{1}{\pi^{2} \epsilon_{0}}\left[\frac{m k_{B} T_{-2}^{2}}{T_{-1}}\right]^{1 / 2} \simeq 4.07 \times 10^{-17} \frac{T_{-2}}{T_{-1}^{1 / 2}} \quad \text { for } \quad 2 \lesssim L / L_{D} \lesssim 7
$$

${ }_{276}$ With a roughly maxwellian core of temperature $T_{c}$ and a dilute halo, we have $T_{-2} / T_{-1}^{1 / 2} \simeq$ ${ }_{277} T_{c}^{1 / 2}$, whence

$$
V_{f}^{2} \simeq 4.07 \times 10^{-17} T_{c}^{1 / 2} \text { for } 2 \lesssim L / L_{D} \lesssim 7
$$

${ }_{278}$ Note that with a Kappa distribution we have

$$
\frac{T_{-2}}{T_{-1}^{1 / 2}}=T^{1 / 2} \frac{(\kappa-3 / 2)^{1 / 2} \Gamma(\kappa)}{\Gamma(\kappa+1 / 2)}
$$

${ }_{279}$ yielding $T_{-2}^{2} / T_{-1} \simeq 0.66 \times T \simeq 0.93 \times T_{-2}$ for $\kappa=4$, so that the temperature measured via

${ }_{280}$ the plateau level is close to that defining the Debye length, similar to a "core" temperature.

${ }_{281}$ However with $\kappa=2$, we have $T_{-2}^{2} / T_{-1} \simeq 0.85 \times T_{-2}$, so that in that case, the plateau 
${ }_{282}$ yields a temperature smaller than the "core" temperature $T_{-2}$ by about $15 \%$; this reflects

${ }_{283}$ the shortage of low-energy particles for kappa distributions with respect to Maxwellians.

Figure 5 shows the levels of the quasi-thermal plateau in a density-core-temperature plane with a dipole antenna made of two colinear $L=2 \mathrm{~m}$ wires, for applications to Parker

${ }_{286}$ Solar Probe/FIELDS. The orange crosses sketch the parameters expected at perihelion ${ }_{287}\left(n \simeq 7000 \mathrm{~cm}^{-3}, T \simeq 10^{6} \mathrm{~K}\right)$. We show the power at both the antenna ports, $V_{f}^{2}$, given by ${ }_{288}$ (36) (left) and at the receiver ports, $V_{r}^{2}$, (right). The temperature shown on the vertical ${ }_{289}$ axis is $T_{-2}^{2} / T_{-1}$, very close to that of the cold Maxwellian when the distribution is a cold ${ }_{290}$ Maxwellian with a suprathermal tail. We have superimposed the approximation (40) as ${ }_{292}$ to a capacitance [Meyer-Vernet and Perche, 1989]

$$
C_{a} \simeq \pi \epsilon_{0} L / \ln \left(L_{D} / a\right)
$$

${ }_{293}$ when $L / L_{D} \gg 1$, so that one deduces from (7)

$$
V_{r}^{2} / V_{f}^{2} \simeq 1 /\left(1+C_{b} / C_{a}\right)^{2}
$$

${ }_{294}$ where $C_{b}$ is the (dipole) load/stray capacitance, which lumps together the receiver input ${ }_{295}$ capacity and that of the antenna erecting mechanism, including the capacity between ${ }_{296}$ the antenna and the spacecraft structure (the so-called base capacity). With $L=2 \mathrm{~m}$, ${ }_{297} a=1.6 \times 10^{-3} \mathrm{~m}$ and $L_{D} \simeq 0.8 \mathrm{~m}$ at 10 solar radii, we have $C_{a} \simeq 8.9 \mathrm{pF}$, whence with ${ }_{298} C_{b}=35 \mathrm{pF}$ (see Table 1 ), $V_{r}^{2} / V_{f}^{2} \simeq 0.04$. This yields a plateau level at the receiver ports ${ }_{299} V_{r}^{2} \simeq 1.7 \times 10^{-15} \mathrm{~V}^{2} \mathrm{~Hz}^{-1}$, which requires a receiver sensitivity of at least a few tens $\mathrm{nV}$ $\mathrm{Hz}^{-1 / 2}$. These evaluations assume that the ion (Section 2.8) and shot noise contributions 
324 collinear - making an angle of $120^{\circ}$ - with a significant gap between them [Gurnett et al.,

${ }_{344}$ [Moncuquet et al., 2005, 2006b]. 


\subsection{Electron Total Density and Kinetic temperature}

The most basic properties of a particle velocity distribution are the total electron density and kinetic temperature. In general, these properties are obtained by fitting the QTN spectrum to the data, except in the ideal case of an antenna much longer than the Debye length immersed in an isotropic plasma, for which these properties are revealed without any fitting (Figure 2). Indeed, the QTN spectral peak reveals the plasma frequency -

351 and (33) yield

$$
V_{f}^{2} \simeq \frac{32 m \omega_{p}^{2}}{4 \pi \epsilon_{0}} \int_{0}^{\infty} d v v f(v) \int_{\omega / v}^{\infty} d k F(k) / k^{3} \quad \text { for } f \gg f_{p}
$$

352 If $f L /\left(f_{p} L_{D}\right) \gg 1$, substituting $F(k) \simeq \pi /(4 k L)$ in (45) yields

$$
V_{f}^{2} \simeq f_{p}^{2} k_{B} T /\left(\pi \epsilon_{0} L f^{3}\right)
$$

${ }_{353}$ The high-frequency QTN is directly proportional to the kinetic temperature $T \equiv T_{2}$ ${ }_{354}$ whatever the shape of the velocity distribution. This $f^{-3}$ spectrum is clearly seen on 355 Figure 2. Note that $V_{f}^{2}$ is deduced from the power measured $V_{r}^{2}$ at the receiver ports by 356 using (44) with the dipole antenna capacitance in this high frequency range

$$
C_{a} \simeq \pi \epsilon_{0} L /[\ln (L / a)-1]
$$

${ }_{357}$ Such an observation, however, requires that no radioemission perturbs the spectrum. This ${ }_{358}$ can be seen on Figure 7 which shows a radio spectrogram from WIND/WAVES acquired 359 in the solar wind during the detection of intense solar radioemissions. The power density ${ }_{360}$ below $f_{p}$ (revealed by the line of increased power), produced by the plasma QTN, remains 
369 resolution, as shown in the following section.

\subsection{Hot Electrons} kinetic temperature.

.6. Hot Electrons tenna, whence $f_{p}$, change rapidly with time).

unperturbed and can still be used to deduce the cold electron temperature (see Section 2.4 ), but the power is strongly perturbed above $f_{p}$ and cannot be used for measuring the

The total electron density can be deduced from the location of the plasma frequency peak. However, this peak can be shifted from $f_{p}$ by several effects. First of all, even in the absence of Doppler-shifts, the spectral peak may be slightly shifted from $f_{p}$, by an amount which depends on the antenna length (via the factor $F(k)$ in (9) as shown by Meyer-Vernet and Perche [1989]), on the distribution of hot electrons and on the frequency and time

Since electrons interact with waves of phase speed equal to their proper speed, and the phase speed of Langmuir waves $\omega / k \rightarrow \infty$ when $\omega \rightarrow \omega_{p}$, the shape of the plasma frequency peak is determined by high speed electrons; the closer the frequency to $f_{p}$, the higher the speed of electrons producing the power. This property is illustrated by the extreme behaviour of the "square" velocity distribution $f(v) \propto \mathrm{H}\left(v_{0}-v\right)$, the Heaviside step function, which produces no QTN peak at $f_{p}$ because of the lack of electrons having the proper speed to interact with the waves [Chateau and Meyer-Vernet, 1989].

Detecting very high energy electrons via QTN spectroscopy therefore requires two receiver properties which may be difficult to conciliate: a high frequency resolution (to measure accurately the peak shape), and a high temporal resolution (because the spacecraft/plasma relative motion and the turbulence make the electron density near the an- 
To illustrate this point, consider an electron velocity distribution made of a sum of 383 isotropic distributions $f_{i}$. From $(33)$, we have

$$
\left\langle\rho^{2}(\mathbf{k}, \omega)\right\rangle^{(0)}=\frac{(2 \pi e)^{2}}{k} \sum_{i} B_{i}(k)
$$

384 where

$$
B_{i}(k)=\int_{\omega / k}^{\infty} d v v f_{i}(v)
$$

${ }_{385}$ The imaginary part of the longitudinal dielectic function $\epsilon_{L}(k, \omega)$ is then

$$
\operatorname{Im}\left(\epsilon_{L}\right)=\frac{2 \pi^{2} e^{2} \omega}{\epsilon_{0} m k^{3}} \sum_{i} f_{i}(\omega / k) \equiv I_{L}
$$

${ }_{386}$ For $\omega / k v \gg 1$, the real part of $\epsilon_{L}$ can be approximated by

$$
\operatorname{Re}\left(\epsilon_{L}\right) \simeq 1-\left(\omega_{p}^{2} / \omega^{2}\right)\left(1+k^{2}\left\langle v^{2}\right\rangle / \omega^{2}\right) \equiv R_{L}
$$

${ }_{387}$ whose nearly real zero is

$$
k_{L} \simeq \omega\left(\omega^{2} / \omega_{p}^{2}-1\right)^{1 / 2} /\left(\left\langle v^{2}\right\rangle\right)^{1 / 2}
$$

${ }_{388}$ The contribution of this zero to the integral (9) (using (2)) can be calculated by writing

${ }_{389} R_{L} \simeq\left(k-k_{L}\right) \partial R_{L} / \partial k$ for $k \simeq k_{L}$ at $f \simeq f_{p}$, with from $(51)$

$$
\partial R_{L} / \partial k \simeq-2 k_{L}\left\langle v^{2}\right\rangle / \omega_{p}^{2} \equiv-R_{L}^{\prime}
$$

390 Therefore (2) and (9) yield for $f=f_{p}+\Delta f$ with $\Delta f \ll f_{p}$ 


$$
V_{f}^{2} \simeq \frac{8}{\pi \epsilon_{0}^{2}} \frac{F\left(k_{L} L\right)}{k_{L}^{2}} \frac{\left\langle\rho^{2}\left(k_{L}, \omega_{p}\right)\right\rangle^{(0)}}{R_{L}^{\prime}\left(k_{L}, \omega_{p}\right) I_{L}\left(k_{L}, \omega_{p}\right)}
$$

391 Substituting (48), (50) and (53) yields the shape of the QTN peak

$$
V_{f}^{2} \simeq \frac{8 m v_{p h} F\left(\omega_{p} L / v_{p h}\right)}{\pi \epsilon_{0}\left\langle v^{2}\right\rangle}\left[\frac{\sum_{i} \int_{v_{p h}}^{\infty} d v v f_{i}(v)}{\sum_{i} f_{i}\left(v_{p h}\right)}\right]
$$

392 where

$$
v_{p h} \simeq \omega_{p} / k_{L} \simeq\left(\left\langle v^{2}\right\rangle f_{p} / 2 \Delta f\right)^{1 / 2} \simeq\left[\left(3 k_{B} T / 2 m\right)\left(f_{p} / \Delta f\right)\right]^{1 / 2}
$$

${ }_{393}$ and in (55)-(56), $\left\langle v^{2}\right\rangle$ and $T$ (the kinetic temperature) concern the whole velocity dis394 tribution. One sees from (55) that the noise at frequency $f=f_{p}+\Delta f$ is produced by 395 electrons moving faster than $v_{p h}$ given by (56). The detailed shape of the peak is governed 396 by the value of $\omega_{p} L / v_{p h}$ (determining $F$ given by (10)) and by the electron population $f_{i}$ 397 that dominates the bracket in (55). If $f(v) \simeq f_{i}(v)$ for $v \geqslant v_{p h}$, the same population $i$ 398 dominates both the numerator and the denominator of this bracket, which simplifies to

$$
[\ldots] \simeq \int_{v_{p h}}^{\infty} d v v f_{i}(v) / f_{i}\left(v_{p h}\right)
$$

399 Hence in that case the amplitude and shape of the QTN peak depend only on the shape ${ }_{400}$ of the component $i$ of the distribution and not of its relative density.

${ }_{401}$ Consider the case when at speeds $v \gtrsim v_{p h}$, the distribution can be approximated by a hot ${ }_{402}$ Kappa halo given by (27) with density $n_{h}$ and temperature $T_{h}=\left(m v_{0}^{2} / 2 k_{B}\right)[\kappa /(\kappa-3 / 2)]$.

${ }_{403}$ With for example $n_{h} / n=0.05, T_{h} / T=10$ and $\kappa=5$, one can verify that this holds when ${ }_{404} \Delta f / f_{p} \lesssim 0.1$ (when the core is Maxwellian). The bracket in (55) then reduces to 


$$
[\ldots] \simeq \frac{k_{B} T_{h}}{m} \frac{\kappa-3 / 2}{\kappa}\left[1+\frac{3 T}{4 T_{h}(\kappa-3 / 2)} \frac{f_{p}}{\Delta f}\right]
$$

${ }_{405}$ When $\Delta f / f_{p}>3 T /\left[4 T_{h}(\kappa-3 / 2)\right]$, which holds with the above parameters when $\Delta f / f_{p}>$ 406 0.02, the bracket in (58) reduces to unity in order of magnitude, so that (55) and (58) 407 yield

$$
V_{f}^{2} \simeq \frac{8 m v_{p h} F\left(\omega_{p} L / v_{p h}\right)}{3 \pi \epsilon_{0}} \frac{T_{h}}{T} \frac{\kappa-3 / 2}{\kappa}
$$

${ }_{408}$ An interesting property emerges from (59). In this exterior part of the peak $(0.02<$ ${ }_{409} \Delta f / f_{p}<0.1$ with the above parameters), the power is not only independent of the ${ }_{410}$ density of the halo, it is also similar for a Kappa halo and a Maxwellian halo $(\kappa \rightarrow \infty)$, ${ }_{411}$ if they have similar most probable speeds $\left(v_{0}=\left[(\kappa-3 / 2) \times T_{h} / \kappa\right]^{1 / 2}\right.$, from (28)). Let us ${ }_{412}$ estimate the amplitude of the peak in this frequency range. We have from (56) and (26)

$$
\frac{\omega_{p} L}{v_{p h}} \simeq \frac{L}{L_{D}}\left(\frac{T_{-2}}{T}\right)^{1 / 2}\left(\frac{2 \Delta f}{3 f_{p}}\right)^{1 / 2}
$$

${ }_{413}$ For $\Delta f / f_{p} \simeq 0.05$ (which lies in the range determined above) and $L / L_{D}<5$, (60) yields ${ }_{414} \omega_{p} L / v_{p h}<1$, so that $F\left(\omega_{p} L / v_{p h}\right) \simeq\left(\omega_{p} L / v_{p h}\right)^{2} / 24$ and (59) and (56) yield $V_{f}^{2} / T^{1 / 2} \simeq$ ${ }_{415} 5 \times 10^{-16}$ for $L / L_{D} \simeq 5$.

${ }_{416}$ Closer to $f_{p}\left(\Delta f / f_{p}<0.02\right.$ in our example $)$, the right-hand side term of the bracket in ${ }_{417}$ (58) becomes dominant, which means that $v_{p h}$ is such that the electrons producing the noise are in the speed range where the hot kappa distribution (27) behaves as a power ${ }_{419}$ law velocity distribution $f(v) \propto v^{-p}$ with $p=2(\kappa+1)$. In that case, (58) yields

$$
[\ldots] \simeq \frac{3 k_{B} T}{4 m \kappa} \frac{f_{p}}{\Delta f}
$$


${ }_{420}$ which no longer depends on $T_{h}$ (nor $n_{h}$ ), and we get from (55), (56), (11), (60) and (61)

$$
V_{f}^{2} \simeq \frac{\left(m k_{B} T\right)^{1 / 2}}{6^{3 / 2} \pi \epsilon_{0} \kappa} \frac{T_{-2}}{T} \frac{L^{2}}{L_{D}^{2}}\left(\frac{f_{p}}{\Delta f}\right)^{1 / 2}
$$

${ }_{421}$ so that the power increases strongly very close to $f_{p}$, as $\left(f_{p} / \Delta f\right)^{1 / 2}$, yielding a peak located ${ }_{422}$ at $f_{p}$. This contrasts with the behavior for a Maxwellian halo $(\kappa \rightarrow \infty)$, in which case ${ }_{423}$ the bracket (58) equals $k_{B} T_{h} / m$, so that (55) yields

$$
V_{f}^{2} \simeq \frac{8 m T_{h} v_{p h}}{3 \pi \epsilon_{0} T} F\left(\omega_{p} L / v_{p h}\right)
$$

${ }_{424}$ Equation (63) shows that when $\Delta f \rightarrow 0\left(v_{p h} \rightarrow \infty\right), V_{f}^{2} \rightarrow 0$, so that the noise peak ${ }_{425}$ is shifted above $f_{p}$ [Meyer-Vernet and Perche, 1989], at the value of $\Delta f$ for which ${ }_{426} v_{p h} F\left(\omega_{p} L / v_{p h}\right)$ is maximum.

${ }_{427}$ Let us use these results to determine whether QTN spectroscopy can be used to measure ${ }_{428}$ the solar wind super-halo electrons, which have a nearly isotropic power-law velocity ${ }_{429}$ distribution at energies exceeding $E_{0} \simeq 2 \mathrm{keV}$ [e.g., Wang et al., 2012]. Using $v_{p h}$ given ${ }_{430}$ by (56), we see that these electrons are revealed at frequencies $f_{p}+\Delta f$ with $\Delta f / f_{p}<$ ${ }^{431}(3 / 4) \times T_{\mathrm{eV}} / E_{0} \simeq 4 \times 10^{-3}$ if $T \simeq 10 \mathrm{eV}$. Such an observation also requires that the $f_{p}$ ${ }_{432}$ fluctuations produced by turbulent density fluctuations [e.g., Wang et al., 2012] occuring ${ }_{433}$ during the measurement of the peak do not broaden it by more than $\Delta f$, which imposes ${ }_{434}$ a constraint on the time resolution that may be difficult to conciliate with the frequency ${ }_{435}$ resolution (because of the Nyquist-Shannon theorem). Using (62) with $\kappa=p / 2-1$, we ${ }_{436}$ obtain for $p=7$ and $L / L_{D}=5, V_{f}^{2} / T^{1 / 2} \simeq 10^{-16}\left(f_{p} / \Delta f\right)^{1 / 2}$. For $\Delta f / f_{p} \simeq 4 \times 10^{-3}$, ${ }_{437}$ this yields $V_{f}^{2} / T^{1 / 2} \simeq 1.4 \times 10^{-15} \mathrm{~V}^{2} \mathrm{~Hz}^{-1}$, i.e. about $4 \times 10^{-13} \mathrm{~V}^{2} \mathrm{~Hz}^{-1}$ for $T \simeq 10^{5}$ 
${ }^{438} \quad$ K. Since $(1 / \Delta f) \int_{f_{p}}^{f_{p}+\Delta f} d f\left[f_{p} /\left(f-f_{p}\right)\right]^{1 / 2}=2$, a receiver with this frequency resolution ${ }_{439}$ should mesure twice this power, i.e. $V_{f}^{2} \simeq 10^{-12} \mathrm{~V}^{2} \mathrm{~Hz}^{-1}$.

${ }_{440}$ It is interesting to note that such a very high noise level, corresponding to QTN pro${ }_{441}$ duced by super-halo electrons, could be erroneously interpreted instead as due to plasma 442 instabilities.

\subsection{Flat-top Distributions}

Flat-top distributions are observed in various media, under conditions when all particles ${ }_{444}$ are accelerated up to a similar energy, for example via an electrostatic field present in a ${ }_{445}$ restricted region. Such velocity distributions have been observed in particular in the ${ }_{446}$ Earth's magnetosheath [Feldman et al., 1982], the Earth's magnetotail around magnetic ${ }_{447}$ reconnection regions [Asano et al., 2008], and downstream of strong interplanetary shocks

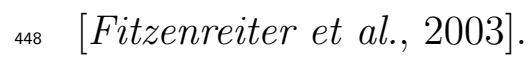


${ }_{460}$ behavior which strongly contrasts with that of a Kappa distribution. Therefore, although

${ }^{461}$ the QTN diagnostics cannot reveal the full flat-top shape, it can nevertheless give a strong

${ }_{462}$ hint of such a shape.

\subsection{Ions}

463

464

${ }_{474}$ by substituting $k \cos \theta=\omega / V$ into (16) that the ion QTN is proportional to the factor ${ }_{475}\left[\sin ^{2}(\omega L / 2 V) /(\omega L / V)\right]^{2}$, which oscillates with frequency and goes to zero at frequencies

${ }_{476}$ that are multiples of $V / L$. Such variations have been observed on WIND/WAVES [Tong ${ }_{477}$ et al., 2015].

${ }_{478}$ An important simplification arises when $\omega L_{D} / V \gg 1$, which holds around the plasma ${ }_{479}$ frequency in the solar wind for PSP at 10 solar radii $\left(\omega_{p} L_{D} / V \simeq 20\right)$. In that case, the 
${ }_{480}$ QTN contribution due to the ions is given by

$$
\begin{array}{ll}
V_{f \text { ions }}^{2} \simeq \frac{8 m_{e} V^{3} \omega_{p}^{2}}{\pi \epsilon_{0} L^{2} \omega^{4}} \sin ^{4}\left(\frac{\omega L}{2 V}\right) & \text { antenna } \| \mathbf{V} \\
V_{\text {fions }}^{2} \simeq \frac{m_{e} V^{2} \omega_{p}^{2}}{\epsilon_{0} L \omega^{3}} & \text { antenna } \perp \mathbf{V}
\end{array}
$$

${ }_{481}$ Comparing with (41), one sees that the ion contribution to the QTN is expected to be ${ }_{482}$ negligible whatever the antenna direction for PSP at perihelion.

\section{QTN in Real Life}

${ }_{483}$ Now that QTN spectroscopy has been admitted in the exclusive club of recognized in

${ }_{484}$ situ measurement techniques, it is essential to ensure that it is not used loosey-goosey, under conditions which might lead to incorrect results. We therefore discuss below some constraints of real life in space which are (or are not) compatible with accurate measurements by QTN spectroscopy, and derive some results that may be useful for practical

${ }_{488}$ applications.

\subsection{Unequal Booms}

${ }_{489}$ When the antenna wires are too thin, they can be broken by dust impacts. This ${ }_{490}$ happened several times for the WIND/WAVES dipole antennas, which now have arms of unequal length. We consider below an antenna made of two wires of respective lengths ${ }_{492} L_{1}$ and $L_{2}$, aligned along the $z$ axis and longer than the gap between them (we do not ${ }_{493}$ consider the effect of a gap since this has been calculated by Meyer-Vernet and Perche $494 \quad[1989])$.

The Fourier transform of the current distribution becomes

$J_{z}(\mathbf{k})=\frac{1}{k_{z}^{2}}\left[\frac{e^{i k_{z} L_{1}}-1}{L_{1}}+\frac{e^{-i k_{z} L_{2}}-1}{L_{2}}\right]$ 
${ }_{496}$ Hence with an isotropic velocity distribution, the QTN is obtained by replacing in (9)

${ }_{497} F(k L)$ by $G\left(k L_{1}, k L_{2}\right)$ given by

$$
\begin{aligned}
G\left(k L_{1}, k L_{2}\right) & =\frac{1}{4 k}\left(\frac{L_{1}+L_{2}}{L_{1} L_{2}}\right)\left\{g\left(k L_{1}\right)+g\left(k L_{2}\right)-g\left[k\left(L_{1}+L_{2}\right)\right]\right\} \\
g(x) & =\frac{\cos x-1}{x}+\operatorname{Si}(\mathrm{x})
\end{aligned}
$$

498

499

$$
\begin{array}{rlrl}
G\left(k L_{1}, k L_{2}\right) & \simeq \frac{k^{2}\left(L_{1}+L_{2}\right)^{2}}{96} & \text { if } & k L_{1}, k L_{2} \ll 1 \\
G\left(k L_{1}, k L_{2}\right) \simeq \frac{\pi}{8 k} \frac{L_{1}+L_{2}}{L_{1} L_{2}} & \text { if } & k L_{1}, k L_{2} \gg 1
\end{array}
$$




\subsection{Fat and/or Biased Antennas}

${ }_{521} \sim\left(2 \pi f_{p}\right)^{-1}\left(\sim\left(2 \pi f_{p h}\right)^{-1}\right.$ for photoelectrons of plasma frequency $\left.f_{p h}\right)$ and a generally

${ }_{522}$ much longer decay time, $\tau_{d}$, due to the discharge of the antenna, the shot noise has a $f^{-2}$

${ }_{523}$ spectrum for $\left(2 \pi \tau_{d}\right)^{-1}<f<f_{p}$ (the squared Fourier transform of a Heaviside function).

${ }_{524}$ In practice, if the electron collection is not much affected by the antenna electric potential

$$
V_{\text {shot }}^{2} \simeq 2\left|I_{e 0}\right| \times e / C_{a}^{2} \omega^{2} \simeq 2 \times 10^{-16}(a / L)\left[\ln \left(L_{D} / a\right)\right]^{2} T_{1}^{1 / 2}\left(f_{p} / f\right)^{2}
$$

${ }_{527}$ for $a<L_{D}<L$ (from Eq.(25) and the formulas by [Meyer-Vernet and Perche, 1989]), ${ }_{528}$ where $C_{a} \simeq\left(i \omega Z_{a}\right)^{-1}$ is the dipole antenna capacitance and $I_{e 0}$ is the electron current ${ }_{529}$ on one antenna arm when $\Phi=0$. Comparing with the expression (40) of the QTN, (71) ${ }_{530} \quad$ yields 


$$
V_{\text {shot }}^{2} / V_{\mathrm{QTN}}^{2} \simeq 4.9 \times \tau(a / L)\left[\ln \left(L_{D} / a\right)\right]^{2}\left(f_{p} / f\right)^{2}
$$

${ }^{531}$ with $\tau=T_{1}^{1 / 2} T_{-1}^{1 / 2} / T_{-2}$, which equals unity for a Maxwellian electron distribution, whereas ${ }_{532} \tau \gtrsim 1$ when the distribution has a suprathermal tail; for example, with a Kappa distri${ }_{533}$ bution we have $\tau=(\kappa-1 / 2) /(\kappa-1)$. With the parameters listed in Table 1 and $\tau \simeq 1$, ${ }_{534}$ (72) yields $V_{\text {shot }}^{2} / V_{\mathrm{QTN}}^{2} \simeq 0.4 \times\left(f_{p} / f\right)^{2}$ for $\mathrm{SO}$ and $V_{\text {shot }}^{2} / V_{\mathrm{QTN}}^{2} \simeq 0.14 \times\left(f_{p} / f\right)^{2}$ for PSP ${ }_{535}$ at perihelion. As noted above, these results assume the frequency to be smaller than $f_{p}$ ${ }_{536}$ and to exceed the inverse of the decay time $\tau_{d} \simeq R C_{a}$ of the antenna potential pulses pro${ }_{537}$ duced by electron impacts and emission, so that these pulses are roughly step-like; here $R$ is the low-frequency antenna resistance due to its discharge by photoelectron emission ${ }_{540}$ by the factor $\omega \tau_{d}$.

${ }_{541}$ The expression (71) of the shot noise also assumes both that the electron collection ${ }_{542}$ is not much affected by the antenna electric potential $\Phi$, and that this potential is the floating potential for which the electron collection current is mainly balanced by the ${ }_{544}$ photoelectron emission current or by the ion current if the latter is larger. This may not ${ }_{545}$ be the case if the antenna is biased since in that case the change in antenna potential ${ }_{546}$ may change significantly the number of elementary charges transferred from and to the antennas, whereas the bias current, $I_{b}$, also contributes to the shot noise. Indeed, since each individual charge transfer to the antenna contributes additively to the shot noise, positive and negative current pulses do not cancel out and they all contribute to the 550 fluctuations. 
Let us first consider the case when the ambient medium is the solar wind. In this case,

$$
I_{p h} \simeq I_{p h 0} \times e^{-\Phi / T_{p h(\mathrm{eV})}}
$$

balances the collected electron current

$$
I_{e} \simeq I_{e 0} \times\left(1+\Phi / T_{e(\mathrm{eV})}\right)
$$

${ }_{556}$ where we have assumed Maxwellian distributions with $\Phi / T_{e(\mathrm{eV})} \ll 1$ since $T_{p h} \ll T_{e}$ ${ }_{557}$ [Whipple, 1981]. In that case, the shot noise $(71)$ is increased by the factor $I_{e} / I_{e 0} \simeq$ ${ }_{558}\left(1+\Phi / T_{e(\mathrm{eV})}\right)$. This result also holds in presence of secondary electron emission $I_{\text {sec }}$ since 559 in that case $\left|I_{\text {sec }}\right|+\left|I_{p h}\right|=\left|I_{e}\right|$ (neglecting the smaller ion current). Note that we have ${ }_{562} 1 /(2 \pi f)$.

$$
V_{\text {shot }}^{2} \simeq\left(\left|I_{e}\right|+\left|I_{p h}\right|+\left|I_{b}\right|\right) e / C_{a}^{2} \omega^{2}
$$

565

since it is proportional to the total number of elementary charges transferred from or to the antenna per time unit, and the contribution of the bias current to the shot noise is 


$$
V_{\text {shot }}^{2} \simeq 2 e \times \operatorname{Max}\left(\left|I_{p h}\right|,\left|I_{e}\right|\right) / C_{a}^{2} \omega^{2}
$$

59 Consider the case when, due to the bias, the antenna potential becomes much smaller than both $T_{p h(\mathrm{eV})}$ and $T_{e(\mathrm{eV})}$, so that $\left|I_{p h}\right| \simeq\left|I_{p h 0}\right|$ and $\left|I_{e}\right| \simeq\left|I_{e 0}\right| \ll\left|I_{p h}\right|$. In that case (76) shows that the bias increases the shot noise (71) by the approximate factor $\left|I_{p h 0} / I_{e 0}\right|$, which amounts to about one order of magnitude in the solar wind; such a bias would make the shot noise largely dominant over the QTN for SO and of the same order of magnitude as the QTN for PSP. On the other hand, biasing the antenna in order to increase its positive potential $\Phi$ would increase the shot noise by a smaller factor. Note that the above estimates assume the antenna photoelectron current to be given by (73), even at small heliocentric distances because - contrary to the spacecraft PSP [Ergun et al., 2010] - the antennas, whose radius is smaller than the photoelectron Debye length, are not expected to be surrounded by a potential barrier reflecting the emitted photoelectrons.

Consider now dense planetary environments, when the plasma ion current dominates the photoelectron current. In that case, since $\left|I_{i 0} / I_{e 0}\right| \simeq\left(m_{e} T_{1 i} / m_{i} T_{1 e}\right)^{1 / 2} \ll 1$, the antenna potential floats to a negative value of order a few times the plasma electron temperature in order to decrease the plasma electron current $I_{e} \simeq I_{e 0} \times e^{-|\Phi| / T_{e(\mathrm{eV})}}$ sufficiently to balance the plasma ion current given by $I_{i} \simeq I_{i 0} \times\left(1+|\Phi| / T_{i(\mathrm{eV})}\right)$ for $\Phi / T_{i(\mathrm{eV})} \ll 1[$ Whipple, 1981]. In that case, the shot noise $(71)$ is decreased by the factor $I_{e} / I_{e 0} \simeq e^{-|\Phi| / T_{e(\mathrm{eV})}}$.

With a bias current, the shot noise is given by (75)-(76) with $\left|I_{p h}\right|$ replaced by $\left|I_{i}\right|$. In that case, if because of the bias, the potential $|\Phi|$ becomes much smaller than both $T_{i(\mathrm{eV})}$ 


$$
q \simeq 4 \pi \epsilon_{0} r_{d} \Phi_{d}
$$

where $\Phi_{d}$ equals a few times the temperature (in $\mathrm{eV}$ ) of the particles that govern the grains' charging, i.e., photoelectrons in the solar wind or ambient electrons in dense planetary environments. Beware that (77) no longer holds when the grain's size is smaller than the Landau radius (the distance at which the mutual electrostatic energy of two plasma 

antennas.

$$
q / Q \simeq 0.015 \times r_{d(\mu \mathrm{m})}^{-2} v_{d(\mathrm{~km} / \mathrm{s})}^{-3.5} \Phi_{d}
$$

Equation (78) generally yields $q / Q \ll 1$, except for submicron particles moving slowly, for example nanodust that have not yet been accelerated, such as freshly produced nanodust in the solar wind or nanodust in inner planetary magnetospheres.

Let us now compare the number of dust particles affecting the electric antennas in dipole mode for both mechanisms. The rate of passing-by dust particles affecting the antennas exceeds the impact rate on their surface by the large factor $L_{D} / a$, of order of magnitude $10^{4}$ for the cases listed in Table 1 (we do not consider the impacts on the spacecraft, which are generally not efficiently detected in dipole mode). These numbers suggest that the electric noise produced by dust grains passing by the antennas may be worth considering. Such a measurement via a time domain sampler has been discussed by Meuris et al. [1996]. We consider below the possibility of such a measurement via a wave receiver, i.e. the quasi-thermal noise produced by dust grains moving around the

In order to derive order-of-magnitude estimates, we consider a simple case: dust grains of charge $q$ and isotropic velocity distribution $f_{d}(v)$. From Eqs. (2), (9) and (33), their QTN is given by 


$$
V_{f \mathrm{~d}}^{2}=\frac{32 q^{2}}{\epsilon_{0}^{2}} \int_{0}^{\infty} d k \frac{F(k L)}{k^{3}\left|\epsilon_{L}(\mathbf{k}, \omega)\right|^{2}} \int_{\omega / k}^{\infty} d v v f_{d}(v)
$$

627

628

$$
f_{d}(v)=n_{d} \delta\left(v-V_{d}\right) /\left(4 \pi V_{d}^{2}\right)
$$

${ }_{629} n_{d}$ being their number density. Substituting (80) into (79) yields

$$
V_{f \mathrm{~d}}^{2}=\frac{8 n_{d} q^{2}}{\pi \epsilon_{0}^{2} V_{d}} \int_{\omega / V_{d}}^{\infty} d k \frac{F(k L)}{k^{3}\left|\epsilon_{L}(\mathbf{k}, \omega)\right|^{2}}
$$

${ }_{630}$ Since $V_{d}$ is much smaller than the electron thermal speed, we can use the approximation (35) of $\epsilon_{L}$ in the integral (81), which reduces to $\epsilon_{L} \simeq 1$ since $k L_{D} \gg 1$ in the integration 32 range. This yields the QTN of this dust distribution

$$
V_{f \mathrm{~d}}^{2}=\frac{8 n_{d} q^{2} L^{2}}{\pi \epsilon_{0}^{2} V_{d}} \int_{\omega L / V_{d}}^{\infty} d x \frac{F(x)}{x^{3}}
$$

${ }_{633}$ Using the approximations (11) and (12), we deduce in particular

$$
\begin{aligned}
& V_{f \mathrm{~d}}^{2}=\frac{2 n_{d} q^{2} V_{d}^{2}}{3 \epsilon_{0}^{2} L \omega^{3}} \quad \text { for } \omega L / V_{d} \gg 1 \\
& V_{f \mathrm{~d}}^{2}=\frac{n_{d} q^{2} L^{2}}{3 \pi \epsilon_{0}^{2} V_{d}} \ln \left(V_{d} / \omega L\right) \text { for } \omega L / V_{d} \ll 1
\end{aligned}
$$

${ }_{634}$ For example, a concentration $n_{d} \simeq 10^{3} \mathrm{~cm}^{-3}$ of nanodust of radius a few nanometers ${ }_{635}$ moving at $V_{d} \simeq 15 \mathrm{~km} / \mathrm{s}$ relative to the Cassini spacecraft in Enceladus' plume [Hill et ${ }_{636}$ al., 2012] should produce from (83) a QTN power of order of magnitude $V_{f \mathrm{~d}}^{2} \simeq 10^{-10}$ 


\section{Concluding Remarks}

We have provided a number of new tools for implementing QTN spectroscopy in space plasmas, which are generally not in thermal equilibrium and are sometimes dusty, inboard various missions. In particular, we give an exact generic expression of the "cold" electron temperature and of its measurement via the QTN plateau (36); we also give a generic analytical approximation (40) of this plateau valid for practical antenna lengths in space, and provide an application for PSP at perihelion. The QTN plateau level is all the more generic, given that we have proven that it still holds in presence of a magnetic field. We also give new analytical approximations of the QTN peak shape and level in several practical cases, and study the conditions in which the solar wind super halo might be measured by this technique. Concerning flat-top distributions, we suggest a simple method to infer them by comparing the low and high frequency QTN levels. Finally, we give new analytical approximations for the QTN due to ions in the solar wind, and show that this component is expected to be negligible for PSP at perihelion.

In order to adapt the method to various practical situations in space, we have considered antennas made of two wires of different lengths, as occurs on WIND after damaging of the antennas by dust impacts, and suggest a new method for determining separately the lengths of the dipole arms. We also consider fat and/or biased antennas, showing that biasing might considerably increase the shot noise in the solar wind, possibly spoiling QTN measurements. Finally, we have estimated the QTN produced by the motion of dust grains near the antennas, yielding a new method to measure grains when their speed is not high enough for producing significant impact ionization. This result may be applied for detecting nanodust in the Enceladus plume, where the plasma shot noise is expected to 
${ }_{661}$ be small because of the strong electron depletion (due to capture of most plasma electrons 
684

\section{References} New York.

Acknowledgments. The authors thank the CNES and the CNRS for support on the Ulysses/URAP, Wind/WAVES and Cassini/RPWS HF projects, and Mélody Pallu and Mathieu Vachey for their help on data processing for Figure 6. The data presented in this paper can be accessed by contacting the authors.

Abramowitz, M. and I. A. Stegun (1965), Handbook of mathematical functions, Dover,

Alexandrov, A. F., L. S. Bogdankevich and A. A. Rukhadze (1984), Principles of plasma electrodynamics, Springer, Berlin, p. 114. 
Asano, Y. et al. (2008) Electron flat-top distributions around the magnetic reconnection region, J. Geophys. Res., 113, A01207, DOI: 10.1029/2007JA012461.

Auer, S. (2001) Instrumentation, in Interplanetary Dust, ed. E. Grün, B. A. S. Gustafson, S. Dermott and H. Fechtig (Heidelberg:Springer), 385-444.

Bale, S. D. et al. (2016), The FIELDS instrument suite for Solar Probe Plus, Space Sci. Rev., DOI: 10.1007/s11214-016-0244-5.

Chateau, Y. F., N. Meyer-Vernet (1989) Electrostatic Noise in Non-Maxwellian Plasmas: "Flat-Top" Distribution Function, J. Geophys. Res., 94, 15,407-15,414.

Chateau, Y. F., N. Meyer-Vernet (1991) Electrostatic noise in non-Maxwellian plasmas: Generic properties and "kappa" distributions, J. Geophys. Res., 96, 5825-5836.

Couturier, P., S. Hoang, N. Meyer-Vernet and J.-L. Steinberg (1981) Quasi-thermal noise in a stable plasma at rest: theory and observations from ISEE 3, J. Geophys. Res., 86, 11,127-11,138.

Dudík et al. (2017), Non-equilibrium processes in the solar corona, transition region, flares, and solar wind, Solar Physics, xx, xx.

Ergun, R. E. et al. (2010), Spacecraft charging and ion wake formation in the near-Sun environment, Phys. of Plasmas, 17, 072903, doi:10.1063/1.3457484.

Fahr, H. J., M. Heyl (2016) Debye screening under non-equilibrium plasma conditions, Astron. Astrophys., 589, A85.

Fejer, J. A., J. R. Kan (1969) Noise spectrum received by an antenna in a plasma, Radio Sci., 4, 721-728.

Feldman, W. C. et al. (1982) Electron heating within the Earth's bow shock, Phys. Rev. Lett., 49, 199. 
Fitzenreiter, R. J., Ogilvie, K., W., Bale, S. D., Vinãs, A. F. (2003) Modification of the solar wind electron velocity distribution at interplanetary shocks, J. Geophys. Res., 108, 1415-1979, DOI: 10.1029/2003JA009865.

Garrett, H. B. (1981), The charging of spacecraft surfaces, Rev. Geophys. Space Phys., 19, 577-616.

Gurnett, D. A. et al. (2004), The Cassini radio and plasma wave investigation, Space Sci. Rev., 114, 395-463.

Henri, P. et al. (2011), Observations of Langmuir ponderomotive effects using the Solar TErrestrial RElations Observatory spacecraft as a density probe, Physics of Plasmas, $18,082308$.

Hill, T. W. et al. (2012), Charged nanograins in the Enceladus plume, J. Geophys. Res., 117, A05209.

Hoang, S., J.-L. Steinberg, G. Epstein, P. Tilloles, J. Fainberg, R. G. Stone (1980), The low-frequency continuum as observed in the solar wind from ISEE 3 - Thermal electrostatic noise, J. Geophys. Res., 85, 3419-3430.

Hoang, S. et al. (1992), Solar wind thermal electrons in the ecliptic plane between 1 and 4 AU - Preliminary results from the ULYSSES radio receiver, Geophys. Res. Lett., 19, $1295-1298$.

Issautier, K., N. Meyer-Vernet, M. Moncuquet, S. Hoang (1998) Solar wind radial and latitudinal structure of electron density and core temperature from Ulysses thermal noise spectroscopy, J. Geophys. Res., 103, 1969-1979.

Issautier, K., N. Meyer-Vernet, M. Moncuquet, S. Hoang, D. J. McComas (1999) Quasithermal noise in a drifting plasma: Theory and application to solar wind diagnostic on 
Ulysses, J. Geophys. Res., 104, 6691-6704.

Issautier, K., R. M. Skoug, J. T. Gosling, S. P. Gary, D. J. McComas (2001) Solar wind plasma parameters on Ulysses: Detailed comparison between the URAP and SWOOPS experiments, J. Geophys. Res., 106, 15665-15676.

Issautier, K., C. Perche, S. Hoang, C. Lacombe, M. Maksimovic, J.-L. Bougeret, S. Salem (2005) Solar wind electron density and temperature over solar cycle 23: Thermal noise measurements on Wind, Adv. Space Res., 35, 2141-2146.

Issautier, K., G. Le Chat, N. Meyer-Vernet, M. Moncuquet, S. Hoang, R. J. MacDowall, D. J. McComas (2008) Electron properties of high-speed solar wind from polar coronal holes obtained by Ulysses thermal noise spectroscopy: Not so dense, not so hot, Geophys. Res. Lett., 35, CiteID L19101.

Kawasaki, K., S. Inoue, E. Ewang, K. Toyoda, M. Cho (2016), Measurement of electron emission yield by electrons and photons for space aged material, in Proceed. 14th Spacecraft Charging Technology Conf., eds. Hilgers et al., ESA/ESTEC, Noordwijk, NL. Knoll, R., G. Epstein, S. Hoang, G. Huntzinger, J. L. Steinberg, J. Fainberg, F. Grena, R. G. Stone, S. R. Mosier (1978) The 3-dimensional radio mapping experiment /SBH/ on ISEE-C, IEEE Trans., GE-16, 199-204.

Laframboise, J. G. (1966) Theory of spherical and cylindrical Langmuir probes in a collisionless Maxwellian plasma at rest, Report 100, Institute of Aerospace Studies, University of Toronto, Canada.

Lai, S. T., E. Murad, W. J. McNeil (2002) Hazards of hypervelocity impacts on spacecraft, J. Spacecraft Rockets 39 106-114. 
McBride, N. and J. A. M. McDonnell, (1999), Meteoroid impacts on spacecraft: Sporadics, streams, and the 1999 Leonids, Planet. Space Sci. 47, 1005-1013.

Meuris, P., N. Meyer-Vernet and J. F. Lemaire (1996) The detection of dust grains by a wire dipole antenna: the radio dust analyzer, J. Geophys. Res., 101, 24,471-24,477.

Meyer, P., N. Vernet (1974) The impedance of a short antenna in a warm magnetoplasma, Radio Science, 9, 409416.

Meyer, P., N. Vernet (1975) The impedance of a dipole antenna in the ionosphere, 2, Comparison with theory, Radio Science, 10, 529536.

Meyer-Vernet, N., P. Meyer, C. Perche (1977) Noncollisional losses in an inhomogeneous plasma, Phys. Fluids, 20, 536-537.

Meyer-Vernet, N., P. Meyer, C. Perche (1978) Losses due to the inhomogeneous sheath surrounding an antenna in a plasma, Radio Science, 13, 69-73.

Meyer-Vernet, N. (1979) On natural noises detected by antennas in plasmas, J. Geophys. Res., 84, 5373-5377.

Meyer-Vernet, N. et al. (1986a), Plasma diagnosis from thermal noise and limits on dust flux or mass in Comet Giacobini-Zinner, Science, 232, 370374.

Meyer-Vernet, N. et al. (1986b), Physical parameters for hot and cold electron populations in Comet Giacobini-Zinner with the ICE radio experiment, Geophys. Res. Lett., 13, 279282.

Meyer-Vernet, N. and C. Perche (1989) Toolkit for antennae and thermal noise near the plasma frequency, J. Geophys. Res., 94, 2405-2415.

Meyer-Vernet, N., S. Hoang, M. Moncuquet (1993) Bernstein waves in the Io plasma torus: A novel kind of electron temperature sensor, J. Geophys. Res., 98, 21,163-21,176. 
Meyer-Vernet, N. (1993), Aspects of Debye shielding, Am. J. Phys., 61, 249-257.

Meyer-Vernet, N. (1994) On the thermal noise" temperature"in an anisotropic plasma, Geophys. Res. Lett., 21, 397-400.

Meyer-Vernet, N., S. Hoang, K. Issautier, M. Maksimovic, R. Manning, M. Moncuquet, R. Stone (1998), Measuring plasma parameters with thermal noise spectroscopy, in Measurement techniques in space plasmas: fields, Geophys. Monograph Ser., vol. 103, edited by R. Pfaff et al., AGU, Washington DC., pp. 205-210.

Meyer-Vernet, N., S. Hoang, K. Issautier, M. Maksimovic, M. Moncuquet, G. Marcos (2000), Plasma thermal noise: the long wavelength radio limit, in Radioastronomy at long wavelengths, Geophys. Monograph Ser., vol. 119, edited by R. G. Stone et al., AGU, Washington DC., pp. 67-74.

Meyer-Vernet, N. (2001) Large scale structure of planetary environments: the importance of not being Maxwellian, Planet. Space Sci., 49, 247-260.

Meyer-Vernet (2013), On the charge of nanograins in cold environments and Enceladus dust, Icarus, 226, 583-590.

Meyer-Vernet, N., M. Moncuquet, K. Issautier, and P. Schippers (2016), Frequency range of dust detection in space with radio and plasma wave receivers: Theory and application to interplanetary nanodust impacts on Cassini, J. Geophys. Res. Space Physics, 121 doi:10.1002/2016JA023081.

Moncuquet, M., N. Meyer-Vernet, S. Hoang (1995), Dispersion of electrostatic waves in the Io plasma torus and derived electron temperature, J. Geophys. Res., 100, 21697-21708.

Moncuquet, M., N. Meyer-Vernet, S. Hoang, R. J. Forsyth, P. Canu (1997), Detection of Bernstein wave forbidden bands in the Jovian magnetosphere: A new way to measure 
the electron density, J. Geophys. Res., 102, 2373-2380.

Moncuquet, M., A. Lecacheux, N. Meyer-Vernet, B. Cecconi, W. S. Kurth (2005), Quasi thermal noise spectroscopy in the inner magnetosphere of Saturn with Cassini/RPWS: Electron temperatures and density, Geophys. Res. Lett., 32, CiteID L20S02.

Moncuquet, M. et al. (2006a), The radio waves and thermal electrostatic noise spectroscopy (SORBET) experiment on BEPICOLOMBO/MMO/PWI: Scientific objectives and performance, Adv. Space Res., 38, 680-685.

M. Moncuquet, N. Meyer-Vernet, A. Lecacheux, B. Cecconi, and W. S. Kurth (2006b), Quasi Thermal Noise in Bernstein Waves at Saturn. In H. O. Rucker, W. Kurth, and G. Mann, editors, Planetary Radio Emissions VI, p. 93.

Nicholls, D. C., M. A. Dopita, R. S. Sutherland (2012), Resolving the electron temperature discrepancies in HII regions and planetary nebulae: $\kappa$-distributed electrons, Astrophys. J., 752, 148.

Nyquist, H. (1928), Thermal agitation of electric charge in conductors, Phys. Rev., 23, 110.

Ockham, W. of (1324), Summa Totius Logicae, i. 12.

Rostoker, N. (1961), Fluctuations of a plasma, Nucl. Fusion, 1, 101-120.

Saint-Hilaire, P. et al. (2014), The Cubesat Radio Experiment (CURE) and Beyond: Cubesat-based Low Frequency Radio Interferometry, AGU Fall meeting, abstract SH53B-4231.

Salem, C., J.-M. Bosqued, D. E. Larson, A. Mangeney, M. Maksimovic, C. Perche, R. P. Lin, J.-L. Bougeret (2001), Determination of accurate solar wind electron parameters using particle detectors and radio wave receivers, J. Geophys. Res., 106, 21701-21717. 
Schiff, M., L. (1970), Impedance of a short dipole antenna in a warm isotropic plasma, Radio Sci.+, 5, 1489-1496.

Schippers, P., M. Moncuquet, N. Meyer-Vernet, A. Lecacheux (2013), Core electron temperature and density in the innermost Saturn's magnetosphere from HF power spectra analysis on Cassini, J. Geophys. Res., 118, 7170-7180.

Scudder, J., S. Olbert (1979), A theory of local and global processes which affect solar wind electrons: The origin of typical 1 AU velocity distribution functions - Steady state theory, J. Geophys. Res., 84, 2755.

Scudder, J. D., H. Karimabadi (2013), Ubiquitous non-thermals in astrophysical plasmas: Restating the difficulty of maintaining Maxwellians, Astrophys. J., 770, 26.

Sentman, D. D. (1982), Thermal fluctuations and the diffuse electrostatic emissions, J. Geophys. Res., 8\%, 1455-1472.

Shukla, P. K. and A. A. Mamun (2002) Introduction to dusty plasma physics, IOP Publishing Ltd, Bristol.

Sitenko, A. G. (1967) Electromagnetic fluctuations in plasmas, Academic Press, San Diego, USA.

Swartwout, M. (2013), The First One Hundred CubeSats: A Statistical Look, JoSS, 2, 213-233.

Tong, Y., M. Pulupa, C. S. Salem, S. D. Bale (2015), Investigating WIND quasi-thermal noise spectra between the ion and the electron plasma frequency, AGU Fall Meeting 2015.

Vasyliunas, V. M. (1968), A survey of low-energy electrons in the evening sector of the magnetosphere with Ogo 1 and Ogo 3, J. Geophys. Res., 73, 2839-2885. 
Verheest, F. (1996), Waves and instabilities in dusty space plasmas, Space Sci. Rev., 77, 267-302.

Wang, L. et al. (2012), Quiet-time interplanetary $\simeq 2-20 \mathrm{keV}$ superhalo electrons at solar minimum, Astrophys. J. Lett., 753, L23.

Whipple, E. C. (1981), Potentials of surfaces in space, Rep. Prog. Phys., 44, 1197-1250.

Yoon, P. H. (2014), Electron kappa distribution and quasi-thermal noise, J. Geophys. Res., 119, 7074-7087.

Yoon, P. H., S. Kim, G. S. Choe, Y.-J. Moon (2016), Revised model of the steady-state solar wind halo electron velocity distribution function, Astrophys. J., 826, 204.

Yoon, P. H., J. Hwang, D. K. Shin (2017), Upper hybrid waves and energetic electrons in the radiation belt, J. Geophys. Res., 122, 5365-5376.

Zouganelis, I., Maksimovic, M., Meyer-Vernet, N., Issautier, K., Moncuquet, M., Bale, S. D. (2007), Implemention of the thermal noise spectroscopy on Solar Orbiter, in Proceed. 2nd Solar Orbiter workshop, ESA-SP 641, eds. Marsch, E., Tsinganos, K., Marsden, R., Conroy, L., Noordwijk, Netherlands.

Zouganelis, I. (2008), Measuring suprathermal electron parameters in space plasmas: Implementation of the quasi-thermal noise spectroscopy with kappa distributions using in situ Ulysses/URAP radio measurements in the solar wind, J. Geophys. Res., 113, A08111, doi:10.1029/2007JA012979.

Zouganelis, I. et al. (2010), Measurements of stray antenna capacitance in the STEREO/WAVES instrument: Comparison of the measured voltage spectrum with an antenna electron shot noise model, Radio Sci., 45, RS1005, doi:10.1029/2009RS004194. 
Table 1. Properties of wire dipole antennas (length $L$ of each element, radius $a$, and dipole stray capacitance ${ }^{\mathrm{b}}$ ) used for QTN on ISEE 3-ICE/3D Radio Mapping, Ulysses/URAPa , WIND/WAVES, Cassini/RPWS, MMO-BepiColombo/PWI-WPT, Solar Orbiter (SO)/RPW and Parker Solar Probe (PSP)/FIELDS, and average Debye length in the solar wind at respectively 1 AU (ISEE 3, Ulysses, Wind), 0.3 AU (representative for Bepi-Colombo and SO), 10 $R_{s}$ (closest heliocentric distance of PSP), and in Saturn's magnetosphere at Enceladus' orbit (explored by Cassini).

\begin{tabular}{cccccccc}
\hline Property & ISEE 3 & Ulysses & WIND & Cassini & BepiColombo & SO & PSP \\
\hline$L(\mathrm{~m})$ & 45 & 35 & $50^{\mathrm{c}}$ & 10 & 15 & 6.5 & 2 \\
$a(\mathrm{~mm})$ & 0.2 & 1.1 & 0.2 & 14.3 & 0.21 & 14.2 & 1.59 \\
$C_{b}(\mathrm{pF})$ & 45 & 57 & 20 & 55 & $50 ?^{\mathrm{b}}$ & $?^{\mathrm{b}}$ & $35^{\mathrm{b}}$ \\
$L_{D}(\mathrm{~m})$ & 10 & 10 & 10 & 1 & 5 & 5 & 0.8 \\
\hline
\end{tabular}

a For Ulysses antennas (tapes of length $L$, width $5 \mathrm{~mm}$ and thickness $0.04 \mathrm{~mm}$ ), the radius is that of the cylinder having the same capacitance.

b The base capacitance can be measured accurately only after the antennas have been extended on the spacecraft in space.

c The length indicated holds at the times when Figs 6 and 7 were acquired, i.e. before the antenna wires were broken by dust impacts. 


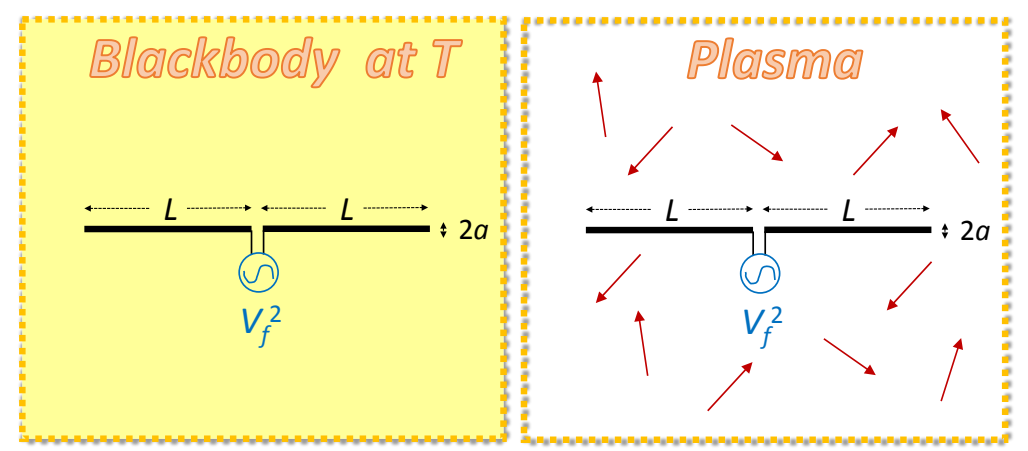

Figure 1. Simple electric antenna (two aligned wires of length $L$ and radius $a$ ) immersed in blackbody radiation (left) and in a plasma (right). When $L_{D} \ll L \ll \lambda=c / f$ ( $L_{D}$ is the plasma Debye length, $c$ the speed of lignt and $f$ the frequency), the antenna resistances are respectively $R_{E M}=2 \pi f^{2} L^{2} /\left(3 \epsilon_{0} c^{3}\right)$ (left) and $R_{P} \simeq(2 / \pi)^{-1 / 2}\left(8 \pi \epsilon_{0} f_{p} L\right)^{-1}$ just below the plasma frequency $f_{p}$ peak, and $R_{P} \simeq f_{p}^{2}\left(4 \pi \epsilon_{0} f^{3} L\right)^{-1}$ for $f \gg f_{p}$ (right). 


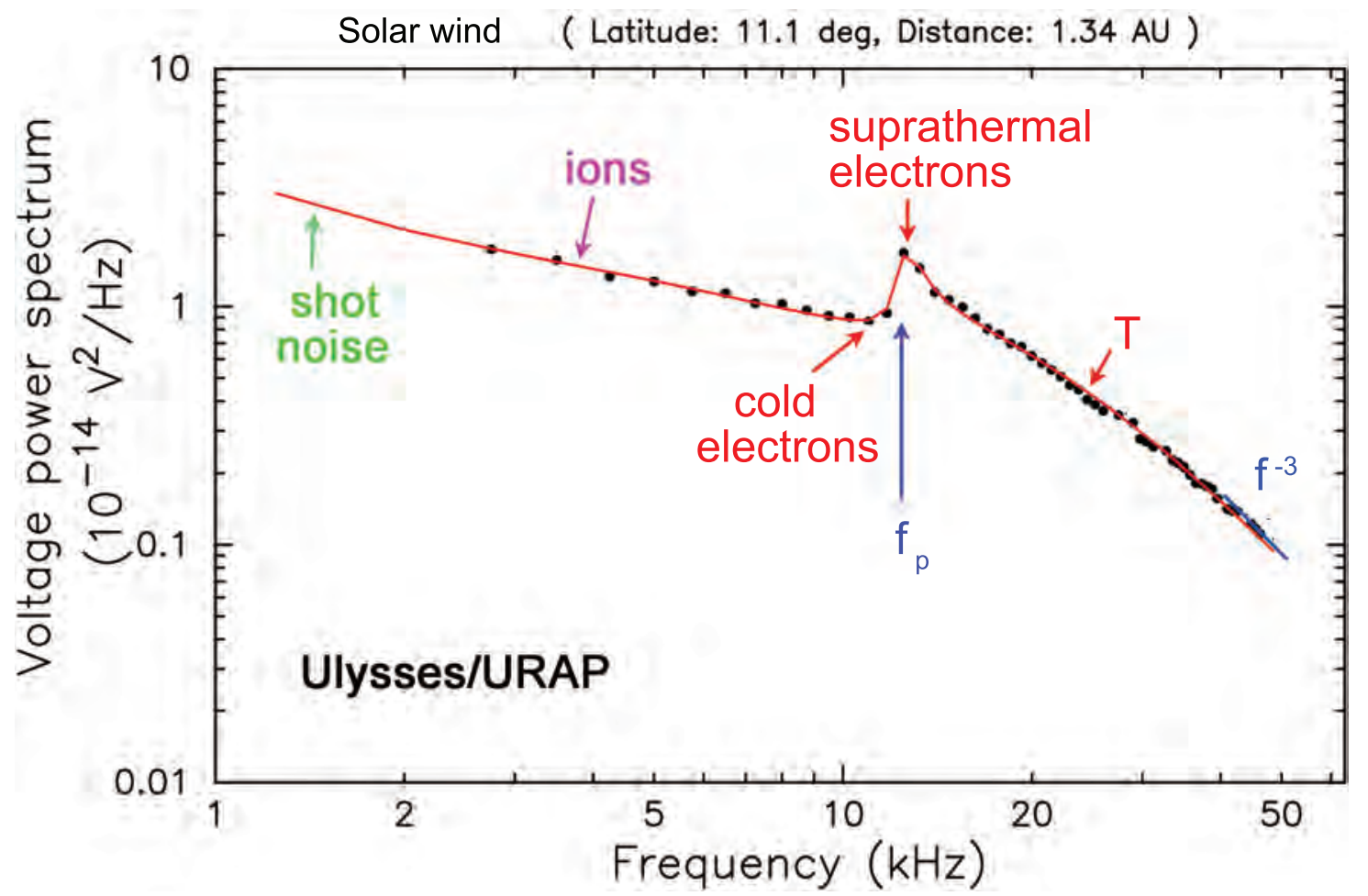

Figure 2. Example of QTN spectrum $\left(V_{r}^{2}\right.$, measured at the receiver's ports) with a wire dipole antenna in a weakly magnetized plasma (Ulysses/URAP data in the solar wind). The main plasma parameters that can be deduced are indicated. Fitted electron parameters, assuming an electron velocity distribution made of a sum of a cold and a hot maxwellian are: $n=1.8 \times 10^{6}$ $\mathrm{m}^{-3}, T_{c}=1.3 \times 10^{5} \mathrm{~K}, T_{h} / T_{c}=8, n_{h} / n_{c}=0.04$ (with an accuracy of $1 \%$ on $n$ and $7 \%$ on $\mathrm{T}$ ). Note that for a quick diagnostics, one can deduce the total electron density from the $f_{p}$ peak, the cold electron temperature from (41) and the kinetic electron temperature from (46), using $V_{f}^{2}$ caculated via (44) with $C_{a}$ from (43) and (47) at respectively low and high frequencies. 


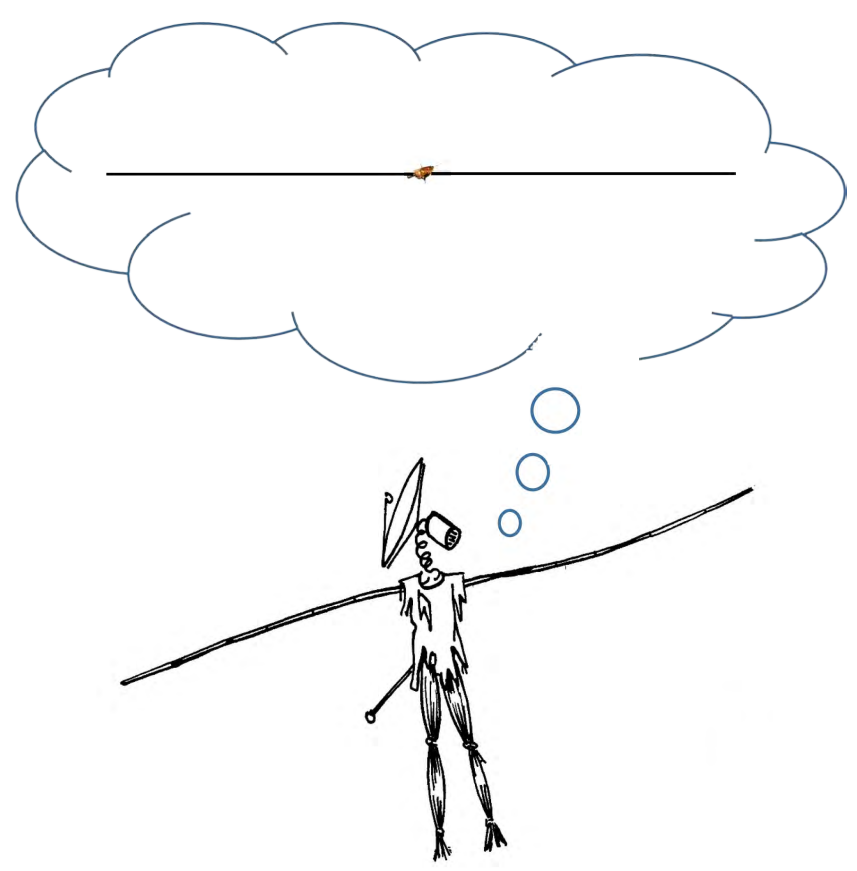

Figure 3. Unfortunately, the strawman payload of the space agencies (bottom) is different from the ideal case for QTN spectroscopy (top), when the spacecraft size is much smaller than the antenna length and the antennas are thin, symmetrical and unbiased - as for ISEE 3 and Ulysses (shown to scale between the antenna wires). Drawing by François Meyer. 


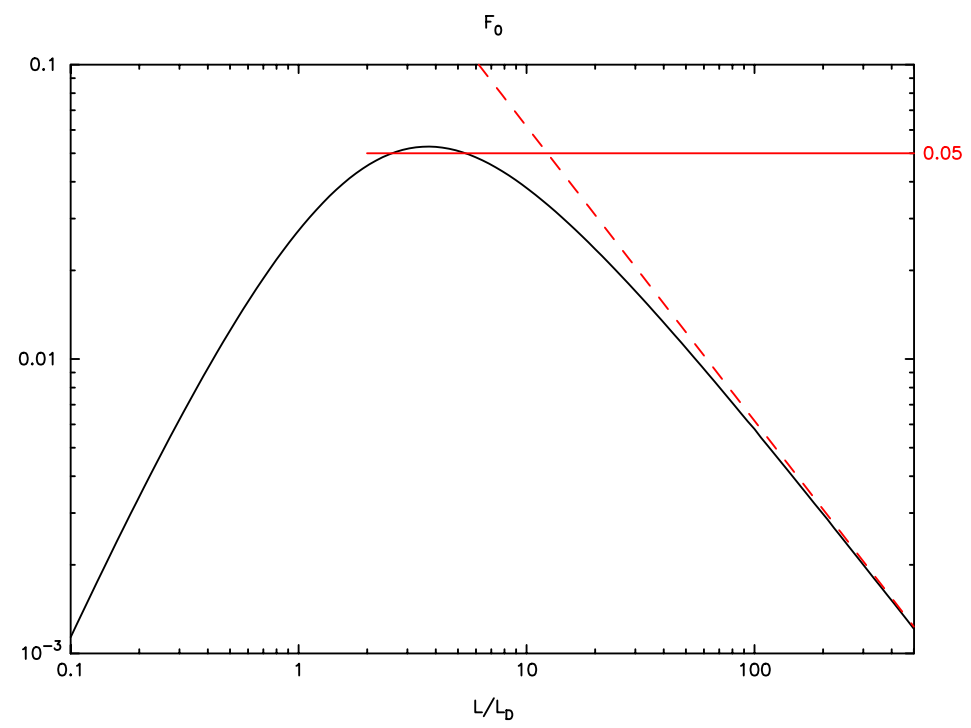

Figure 4. Function $F_{0}\left(L / L_{D}\right)$ given by (37). Multiplying $F_{0}$ by the factor $2^{7 / 2}\left(m k_{B}\right)^{1 / 2} /\left(\pi^{3 / 2} \epsilon_{0}\right) \simeq 8.14 \times 10^{-16}$ yields the QTN plateau $V_{f}^{2}$ normalized to $\left[T_{-2} / T_{-1}^{1 / 2}\right]$, close to the square root of the "cold" temperature (in $\mathrm{V}^{2} \mathrm{~Hz}^{-1} \mathrm{~K}^{-1 / 2}$ ) (see Eq.(36)). The dashed red line shows the approximation (38) (valid for very long antennas); the solid red line shows the approximation $F_{0} \simeq 0.05$ (valid for intermediate lengths). 

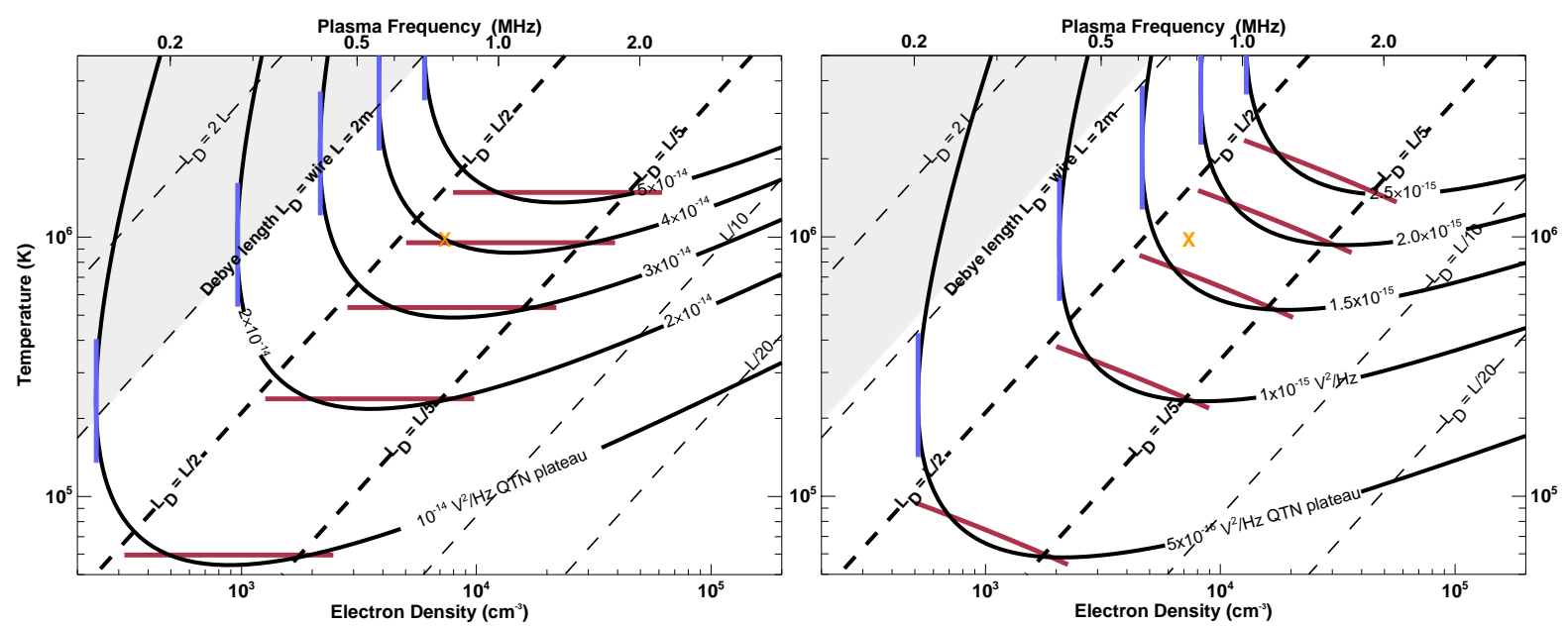

Figure 5. QTN plateau level in $\mathrm{V}^{2} \mathrm{~Hz}^{-1}$ with the wire dipole antenna of PSP/FIELDS $(L=2$ $\mathrm{m}$ ) in a density/temperature plane, with the approximation (40) superimposed as red bars. The power is calculated at both the antenna ports (left, from (36)) and the receiver ports (right, deduced via (7)). The variation in antenna capacitance (43) with $L_{D}$ makes $V_{r}^{2} / V_{f}^{2}$ vary with the electron density, so that the horizontal lines (left) become inclined (right). The orange cross sketches the density and temperature expected for PSP near perihelion. The range $L / L_{D}<1$, in which QTN spectroscopy become ineffective is shown in grey; the blue vertical lines show the regions in which the QTN plateau level becomes weakly dependent of temperature, making this derivation difficult. 

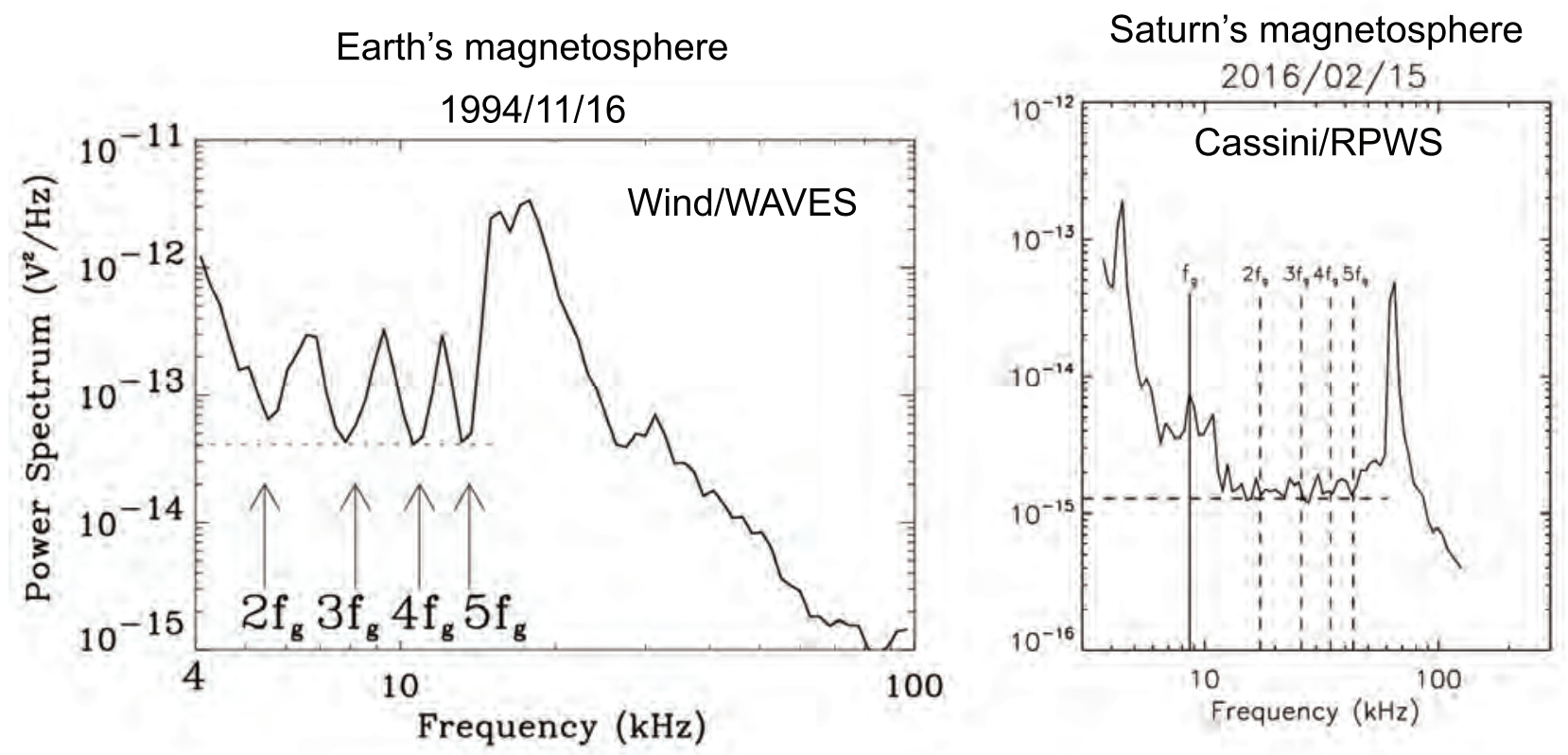

Figure 6. Two examples of quasi-thermal noise spectra $\left(V_{r}^{2}\right.$, measured at the receiver ports $)$ in magnetized plasmas, showing a plateau of minima (dashed horizontal line) at the gyroharmonics $n f_{g}$. Left-hand side: Wind/WAVES data in the Earth's magnetosphere at 8 Earth's radii. Righthand side: Cassini/RPWS data in Saturn's magnetosphere at 4 Saturn's radii. 


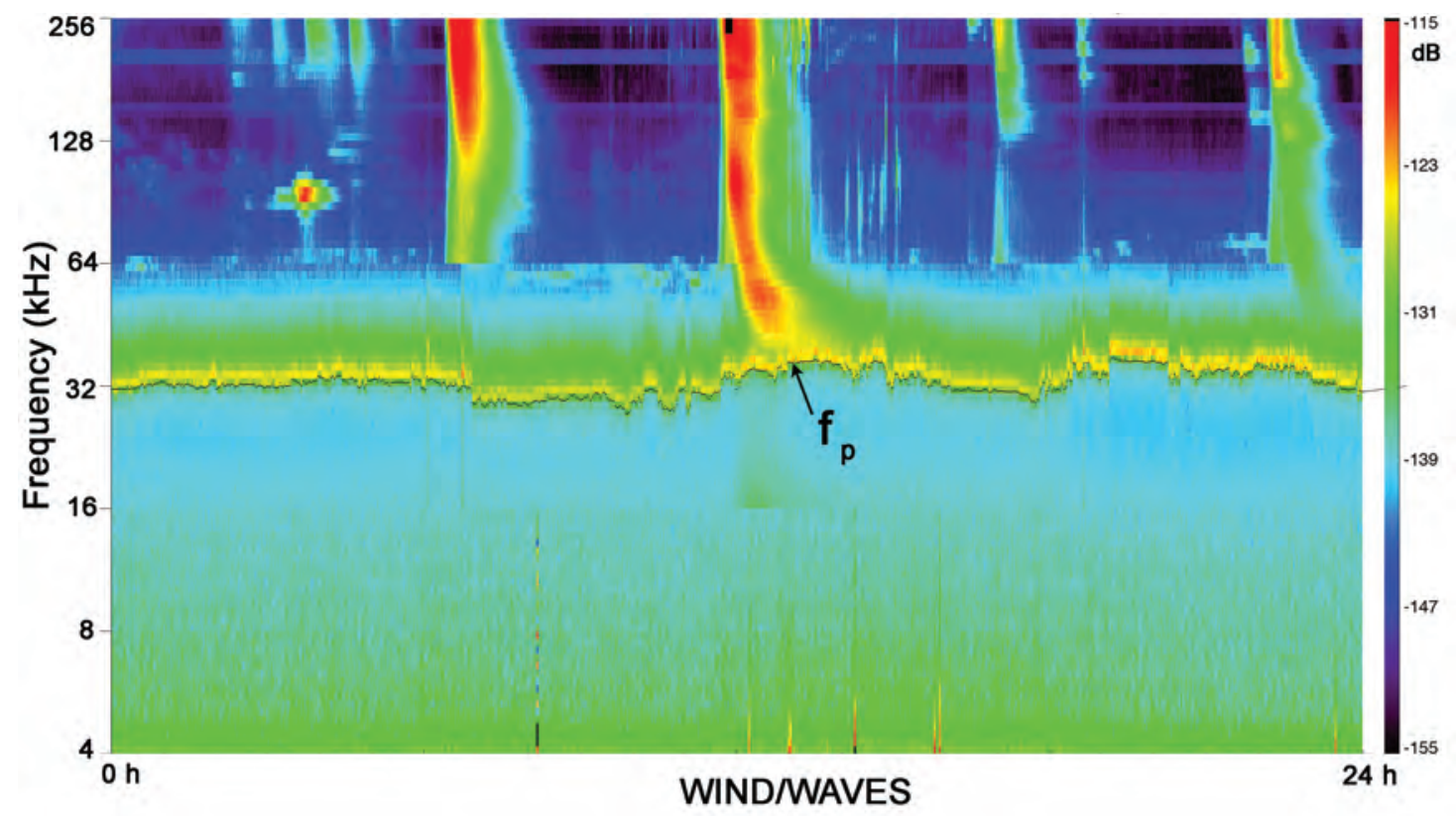

Figure 7. Radio spectrogram from WIND/WAVES acquired on 5 November 1997 in the solar wind, showing solar radioemissions perturbing the plasma QTN above the plasma frequency, whereas the $f_{p}$ line and QTN plateau are not perturbed. The data are plotted as frequency versus time, with the relative intensity coded as indicated in the color bar. 